\title{
Exploiting Trust Degree for Multiple-Antenna User Cooperation
}

\author{
Mingxiong Zhao, Jong Yeol Ryu, Member, IEEE, Jemin Lee, Member, IEEE, \\ Tony Q. S. Quek, Senior Member, IEEE, and Suili Feng, Member, IEEE
}

\begin{abstract}
For a user cooperation system with multiple antennas, we consider a trust degree based cooperation techniques to explore the influence of the trustworthiness between users on the communication systems. For the system with two communication pairs, when one communication pair achieves its quality of service (QoS) requirement, they can help the transmission of the other communication pair according to the trust degree, which quantifies the trustworthiness between users in the cooperation. For given trust degree, we investigate the user cooperation strategies, which include the power allocation and precoder design for various antenna configurations. For SISO and MISO cases, we provide the optimal power allocation and beamformer design that maximize the expected achievable rates while guaranteeing the QoS requirement. For a SIMO case, we resort to semidefinite relaxation (SDR) technique and block coordinate update (BCU) method to solve the corresponding problem, and guarantee the rank-one solutions at each step. For a MIMO case, as MIMO is the generalization of MISO and SIMO, the similarities among their problem structures inspire us to combine the methods from MISO and SIMO together to efficiently tackle MIMO case. Simulation results show that the trust degree information has a great effect on the performance of the user cooperation in terms of the expected achievable rate, and the proposed user cooperation strategies achieve high achievable rates for given trust degree.
\end{abstract}

Index Terms-Trust degree, cooperative transmission, beamforming, power allocation

This work has been supported in part by the Key Discipline Foundation of School of Software of Yunnan University and the Open Foundation of Key Laboratory in Software Engineering of Yunnan Province under Grant No.2017SE203, and the National Natural Science Foundation of China No.61640306, and the National Research Foundation of Korea (NRF) grant funded by the Korea government (MSIP) (No.2017R1C1B2009280) and Institute for Information \& communications Technology Promotion (IITP) grant funded by the Korea government (MSIP) (2014-0-00065, Resilient CyberPhysical Systems Research), and the Guangdong Science and Technology Plan under Grant 2016A010101009.

M. Zhao is with the School of Software, Yunnan University, Kunming, China and also with Key Laboratory in Software Engineering of Yunnan Province. Email: jimmyzmx@gmail.com,mx_zhao@ynu.edu.cn.

J. Y. Ryu is with the Department of Information and Communication Engineering, Gyeongsang National University, Tongyeong, Korea. Email: ryujy18@gmail.com.

J. Lee is with the Department of Information and Communication Engineering, Daegu Gyeongbuk Insitute of Science and Technology (DGIST), Daegu, Korea. Email: jmnlee@dgist.ac.kr.

T. Q. S. Quek is with Singapore University of Technology and Design, Singapore 487372 and also with the Department of Electronics Engineering, Kyung Hee University, Yongin-si, Gyeonggi-do, 17104, Korea. E-mail: tonyquek@sutd.edu.sg.

S. Feng is with the School of Electronics and Information Engineering, South China University of Technology, Guangzhou, China. Email: fengsl@scut.edu.cn.

\section{INTRODUCTION}

The cooperative communications have been introduced to improve the communication reliability and spectral efficiency to satisfy the growing demand for higher data rates in wireless networks. Generally, there are two ways of realizing cooperation: 1) utilizing fixed relay terminals to assist the communication between dedicated sources and their corresponding destinations, 2) allowing the mobile users to help each other as a relay for reliable communication [1]. For utilizing fixed relays, the fixed relays need to be installed in the network, which requires high infrastructure, operation and maintenance costs for the operators [2]. On the other hand, in the user cooperation, relays are the mobile users who have good channel conditions and low traffic demands, and they can help the communications without increasing the cost to the mobile operators [3]. Furthermore, many mobile relays exist in the network, so each relay needs to assist few users only and the average power used by each mobile relay for transmission of signals is much smaller than that of the fixed relays [2]. Due to these advantages, in the cooperative communications, the user cooperation techniques have been intensively investigated [2]-[7].

In the user cooperation, the proximity between users enables their direct communications via device-to-device (D2D) communication. For various communication networks, the techniques for the cooperative D2D communications have been proposed [4]-[7]. For a cellular network, where the cellular and D2D users coexist, the relaying scheme of D2D user to assist the downlink transmission of cellular user was proposed in [4]. The cooperative D2D communication between the femto and macro users of a heterogeneous network (HetNet) was proposed in [5], where the femto user overheard and forwarded the composite of desired and interference signals to improve the signal-to-interference ratio (SINR) of the macro user. In [6], it is shown that mobile terminals can save the energy by exploiting the good channel quality of short range cooperation for WiFi and WiMedia. In the existing literatures [4]-[7], the user cooperation techniques are designed based on their traffic demands and corresponding qualities of physical channels.

However, different from the cooperation using the fixed relays, in the user cooperation, the relationship between users, e.g., trust degree, may affect the performance of user cooperation [8]. The social relationship between users can be an important motivation for participating in cooperative communications. Users would be willing to help each other by 
consuming their own resources if they have close relationship in the social domain. Otherwise, it is not sure whether users will cooperate even though they have the good channels and low traffic demands. Furthermore, some users, generally not with close relationship, may discard the data of the other user during the cooperation due to either the selfish behavior to save its own resource or the malicious purpose to disconnect the communications. Therefore, in the user cooperation, the relationship among users should be taken into account as a key design parameter for the efficient cooperative communications.

Recently, the social relationship has been actively considered in the development of communication strategies such as [9]-[16]. With the consideration of social relationships and physical coupling among users, a social group utility maximization framework was developed to maximize the social group utility, which is a sum of individual utilities weighted by its social ties with other users in [9] and [10]. The social relationship of nodes has also been considered to enhance the performance of D2D communication [11]-[13]. Specifically, social trust and social reciprocity, which were achieved by exchanging the altruistic actions among nodes, were utilized in the D2D relay selection [11], while the socialaware D2D communication architecture was proposed by exploiting social networking characteristics for system design [12]. In [13], a traffic offloading for D2D communications was optimized for given online and offline social relations. The trustworthiness between nodes has also been exploited for the design of efficient cooperation strategies [14]-[16]. In these works, the trust degree, which quantifies a degree of trustworthiness between nodes, was used as one of key design parameters to develop cooperative relay frameworks for the single-input-single-output (SISO) [14] and multipleinput-single-output (MISO) [15] systems. In terms of communication confidentiality, it was also shown that the expected secrecy rate can be increased by exploiting the trust degree of untrustworthy node in [16]. The previous works showed that the trust information can improve the performance of the conventional systems, which are designed based on the physical parameters only.

In this paper, motivated by the strong interests in trust degree, we investigate the user cooperation techniques based on trust degree between two pairs of communication users with multiple antennas, i.e., $\mathrm{Tu}_{1}-\mathrm{Ru}_{1}$ and $\mathrm{Tu}_{2}-\mathrm{Ru}_{2}$. Different from the existing works that consider a simple system model, we consider the cooperation techniques with multiple antennas, including power allocation and precoder design. The transmit user, $\mathrm{Tu}_{2}$, helps $\mathrm{Tu}_{1}$ by forwarding the information of $\mathrm{Tu}_{1}$ when $\mathrm{Tu}_{2}$ has good channel quality to $\mathrm{Ru}_{2}$, enough to guaranteeing its own quality of service (QoS) requirement. The willingness of $\mathrm{Tu}_{2}$ in helping $\mathrm{Tu}_{1}$ is characterized by the trust degree, i.e., $\mathrm{Tu}_{2}$ helps with high probability when trust degree is high. To maximize the expected achievable rate at $\mathrm{Ru}_{1}$, we jointly design the transmission strategies at $\mathrm{Tu}_{1}$ and $\mathrm{Tu}_{2}$ for four different antenna configurations: 1) SISO case where all users equip a single antenna as a special case, 2) MISO case where only $\mathrm{Tu}_{1}$ equips multiple antennas, 3) SIMO case where only $\mathrm{Tu}_{2}$ equips multiple antennas, and 4) MIMO case where both $\mathrm{Tu}_{1}$ and $\mathrm{Tu}_{2}$ equip multiple antennas. For SISO case, we first present an optimal power allocation strategy at $\mathrm{Tu}_{2}$, which maximizes the expected achievable rate at $\mathrm{Ru}_{1}$ while guaranteeing QoS requirement at $\mathrm{Ru}_{2}$. For MISO case, we provide an optimal structure of beamformer at $\mathrm{Tu}_{1}$ as a linear combination of the weighted channel vectors. Then, based on the structure, we obtain the beamformer that maximizes an approximated expected achievable rate as a function of the trust degree and corresponding power allocation at $\mathrm{Tu}_{2}$. For SIMO case, to jointly optimize the beamformers of $\mathrm{Tu}_{2}$, we utilize semidefinite relaxation (SDR) technique and block coordinate update (BCU) method to solve the considered problem, and guarantee the rank-one solutions at each step. Furthermore, for MIMO case, the similarities among the problem structures of MISO, SIMO and MIMO cases inspire us to combine the design of beamformer at $\mathrm{Tu}_{1}$ from MISO and the alternative algorithm from SIMO together to jointly optimize the beamformers at $\mathrm{Tu}_{1}$ and $\mathrm{Tu}_{2}$ to maximize the expected achievable rate at $\mathrm{Ru}_{1}$.

The rest of paper is organized as follows. We first describe the trust degree and system model in Section II In Section III, based on the trust degree, the optimal transmission strategies and beamforming design in terms of the expected achievable rate are derived for four different cases. Numerical results are presented in Section IV and conclusions are drawn in Section $\mathrm{V}$

Notation: In this paper, lowercase and uppercase boldface letters represent vectors and matrices, respectively. The complex conjugate of $x$ is denoted by $\bar{x}$, the hermitian transpose and the trace of $\mathbf{X}$ are denoted by $\mathbf{X}^{\dagger}$ and $\operatorname{tr}(\mathbf{X})$. $\boldsymbol{\Pi}_{\mathbf{X}} \triangleq \mathbf{X}\left(\mathbf{X}^{\dagger} \mathbf{X}\right)^{-1} \mathbf{X}^{\dagger}$ represents the orthogonal projection onto the column space of $\mathbf{X}$, and $\boldsymbol{\Pi}_{\mathbf{X}}^{\perp} \triangleq \mathbf{I}-\boldsymbol{\Pi}_{\mathbf{X}}$ denotes the orthogonal projection onto the orthogonal complement of the column space of $\mathbf{X} . \mathbf{X} \sim \mathcal{C N}(\mathbf{A}, \mathbf{B})$ denotes the elements of $\mathbf{X}$ that follow independent complex Gaussian distribution with mean $\mathbf{A}$ and covariance $\mathbf{B}$.

\section{SySTEM MODEL}

We consider a system with two communication pairs: $\mathrm{Tu}_{1}-$ $\mathrm{Ru}_{1}$ and $\mathrm{Tu}_{2}-\mathrm{Ru}_{2}$, where $\mathrm{Tu}_{1}$ and $\mathrm{Tu}_{2}$ are equipped with $N_{1}$ and $N_{2}$ antennas, respectively, and the receivers have a single antenna. In our system model, if one node achieves its own QoS requirement, it can help the transmission of the other node by using residual resource. Here, we assume that the node decides whether to help the transmission or not based on the trust degree, which measures the trustworthiness between nodes. Without loss of generality, $\mathrm{Tu}_{2}$ helps the transmission of $\mathrm{Tu}_{1}$ according to the trust degree between two user pairs. In the following subsections, we first briefly give an introduction of trust degree, and describe the system model in details.

\section{A. Trust Degree}

With the explosive growth of online social networks such as WeChat and Facebook, a growing number of people are getting involved in online social interactions, and thus, the social relationship has been studied as an important parameter to investigate how the degree of closeness of social relationship between users affects their communication strategies [9]-[11]. 
In the communication networks, the trust degree has been defined as a belief level that one node can put on another node for a certain action according to previous direct or indirect information, obtained from observations of behavior [17], [18]. Hence, in the cooperative communication systems, the trust degree can be interpreted as the degree that reveals how much a node is willing to help the communication of the other node [14], [15]. Similarly, in our system model, the trust degree between $\mathrm{Tu}_{1}$ and $\mathrm{Tu}_{2}, \alpha$, is defined by the probability that $\mathrm{Tu}_{2}$ helps the transmission of $\mathrm{Tu}_{1}$ and thus, $\alpha$ is a value in range of $0 \leq \alpha \leq 1$.

In the previous literatures, the trust degree has been evaluated and quantified by various ways [8], [17]-[19]. The trust degree can be evaluated by the observations of the previous behaviors of the node [17]-[21]. In [20], [21], the trust degree is determined using Bayesian framework. In the Bayesian framework, the trust degree is given by the ratio of the observations of the positive behavior among total observations, where the positive behavior is that the node behaves in the predefined way of the network. Similar to [21], in our cooperative communication systems, the positive behavior is defined by that $\mathrm{Tu}_{2}$ helps the transmission of $\mathrm{Tu}_{1}$ and hence, $\mathrm{Tu}_{1}$ can estimate the trust degree based on the historical observations of the positive behavior of $\mathrm{Tu}_{2}$. The trust degree can also be updated according to new observations. However, when the number of observations is sufficiently large, the trust degree will have ignorable change according to new observation and it will be more like a constant. Therefore, in our system model, we assume that the trust degree is unchanged during the transmission.

In the user cooperative communications, the user may not help the other user's transmission due to either the selfish behavior to save its own resource or the malicious purpose to disconnect the communication of that user. For the case of the malicious purpose, the malicious user lets the other user know that he will help the transmission. However, the malicious user can intercept or drop the data from the other user. Therefore, for the case that the users are not trustworthy, each user designs the transmission strategy based on the trust degree.

\section{B. System Description}

In this paper, we consider the cooperative communication system, where $\mathrm{Tu}_{2}$ can help the transmission of $\mathrm{Tu}_{1}$ if its corresponding receiver $\mathrm{Ru}_{2}$ achieves its QoS, as shown in Fig. 1 In our system, $\mathrm{Tu}_{2}$ decides whether to help the transmission of $\mathrm{Tu}_{1}$ based on the trust degree, $\alpha$, which is defined as the probability that $\mathrm{Tu}_{2}$ cooperates with $\mathrm{Tu}_{1}$ as a relay node. Hence, for given $\alpha$, we design the optimal transmission strategy at $\mathrm{Tu}_{1}$ and the power allocation for cooperation at $\mathrm{Tu}_{2}$ to maximize an expected achievable rate while guaranteeing the $\mathrm{QoS}$ requirement at $\mathrm{Ru}_{2}$. Once $\mathrm{Tu}_{2}$ decides to help the transmission of $\mathrm{Tu}_{1}, \mathrm{Tu}_{2}$ determines the portion of transmission power $\beta$ for relaying information and the portion for its own data transmission. The channels from $\mathrm{Tu}_{1}$ to $\mathrm{Tu}_{2}, \mathrm{Ru}_{1}$, and $\mathrm{Ru}_{2}$ are defined by $\mathbf{H}_{0} \in \mathbb{C}^{N_{2} \times N_{1}}$, $\mathbf{h}_{1} \in \mathbb{C}^{N_{1} \times 1}$, and $\mathbf{h}_{12} \in \mathbb{C}^{N_{1} \times 1}$, respectively, and they

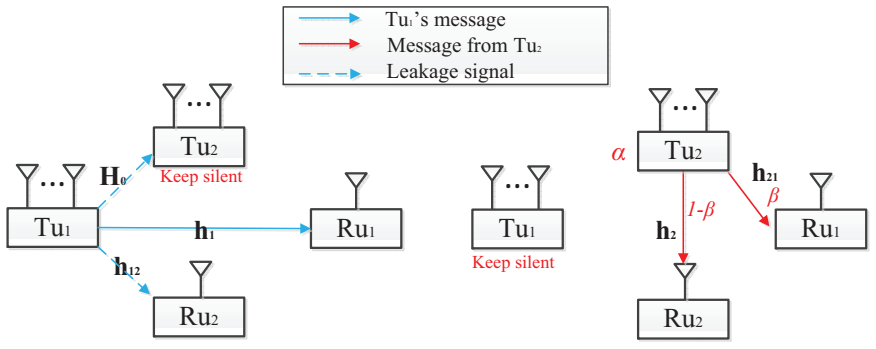

(a) Transmission in time slot $\mathrm{t}_{\mathrm{t}}$

(b) Transmission in time slot $\mathrm{t}$

$\mathrm{t}_{1}=\mathrm{t}_{2}=\mathrm{t}$

Fig. 1. Cooperative communication system

follow a complex Gaussian distribution with zero mean and covariances, $\sigma_{H_{0}}^{2} \mathbf{I}_{N_{2} \times N_{1}}, \sigma_{h_{1}}^{2} \mathbf{I}_{N_{1}}$ and $\sigma_{h_{12}}^{2} \mathbf{I}_{N_{1}}$, respectively. The channels from $\mathrm{Tu}_{2}$ to $\mathrm{Ru}_{1}$, and $\mathrm{Ru}_{2}$ are also defined by $\mathbf{h}_{21} \in \mathbb{C}^{N_{2} \times 1}$ and $\mathbf{h}_{2} \in \mathbb{C}^{N_{2} \times 1}$, which have the covariances $\sigma_{h_{21}}^{2} \mathbf{I}_{N_{2}}$ and $\sigma_{h_{2}}^{2} \mathbf{I}_{N_{2}}$, respectively. Notice that when $\mathrm{Tu}_{2}$ helps the transmission of $\mathrm{Tu}_{1}, \mathrm{Ru}_{1}$ can estimate the channel from $\mathrm{Tu}_{2}, \mathbf{h}_{21}$, and then, $\mathrm{Ru}_{1}$ reports the channel estimation of $\mathbf{h}_{21}$ to $\mathrm{Tu}_{1}$ by feedback channel.

In our system model, the data transmission operates in timedivision mode, where $\mathrm{Tu}_{1}$ and $\mathrm{Tu}_{2}$ transmit their own data at $t_{1}$ and $t_{2}$, respectively, and $t_{1}$ and $t_{2}$ are assigned to be orthogonal with $t_{1}=t_{2}=t$. In the time slot $t_{1}, \mathrm{Tu}_{1}$ transmits the information-carrying symbol $x_{1}$ with $E\left[x_{1} \bar{x}_{1}\right]=1$ to $\mathrm{Ru}_{1}$ and during $t_{1}, \mathrm{Ru}_{2}$ and $\mathrm{Tu}_{2}$ can also listen to $x_{1}$. To efficiently transmit data, $\mathrm{Tu}_{1}$ designs the transmit beamformer, $\mathbf{w}_{1} \in$ $\mathbb{C}^{N_{1} \times 1}$, which satisfies $\mathbf{w}_{1}^{\dagger} \mathbf{w}_{1} \leq P_{1}$ and $P_{1}$ is the maximum transmit budget at $\mathrm{Tu}_{1}$, and uses it for transmission in $t_{1}$. Hence, the received signals at $\mathrm{Ru}_{1}, \mathrm{Ru}_{2}$ and $\mathrm{Tu}_{2}$ in the time slot $t_{1}$ are respectively given by

$$
\begin{aligned}
y_{\mathrm{Ru}_{1}, 1} & =\mathbf{h}_{1}^{\dagger} \mathbf{w}_{1} x_{1}+n_{\mathrm{Ru}_{1}, 1}, \\
y_{\mathrm{Ru}_{2}, 1} & =\mathbf{h}_{12}^{\dagger} \mathbf{w}_{1} x_{1}+n_{\mathrm{Ru}_{2}, 1} \\
\mathbf{y}_{\mathrm{Tu}_{2}} & =\mathbf{H}_{0} \mathbf{w}_{1} x_{1}+\mathbf{n}_{\mathrm{Tu}_{2}},
\end{aligned}
$$

where $n_{\mathrm{Ru}_{1}, 1}$ and $n_{\mathrm{Ru}_{2}, 1}$ represent the additive white Gaussian noise (AWGN) associated with $\mathrm{Ru}_{1}$ and $\mathrm{Ru}_{2}$, which follow the complex Gaussian distribution with zero mean and variance $\sigma^{2}$ (i.e., $\mathcal{C N}\left(0, \sigma^{2}\right)$ ), and $\mathbf{n}_{\mathrm{Tu}_{2}} \in \mathbb{C}^{N_{2} \times 1}$ is the AWGN at $\mathrm{Tu}_{2}$ with $\mathcal{C N}\left(\mathbf{0}, \sigma^{2} \mathbf{I}_{N_{2}}\right)$. We assume that if $\mathrm{Ru}_{2}$ can decode the data from the received signal, $y_{\mathrm{Ru}_{2}, 1}$, in the time slot $t_{1}$, $\mathrm{Ru}_{2}$ can use it as side information for further improving the performance by interference cancellation in $t_{2}$.

In the time slot $t_{2}, \mathrm{Tu}_{2}$ transmits $x_{2}$ with $E\left[x_{2} \bar{x}_{2}\right]=1$ to $\mathrm{Ru}_{2}$. Here, $\mathrm{Tu}_{2}$ has its traffic demand $Q$ and if $\mathrm{Ru}_{2}$ achieves $Q$ by the part of total power budget, $\mathrm{Tu}_{2}$ can help the transmission of $\mathrm{Tu}_{1}$ using residual power. According to the trust degree, $\mathrm{Tu}_{2}$ relays the data of $\mathrm{Tu}_{1}$ to $\mathrm{Ru}_{1}$ via decode-andforward (DF) based relaying with probability $\alpha$ and otherwise, $\mathrm{Tu}_{2}$ transmits its own data only. Thus, the transmitted signal from $\mathrm{Tu}_{2}$ can be represented by

$$
\mathbf{x}_{\mathrm{Tu}_{2}}=\mathbf{w}_{21} x_{1}+\mathbf{w}_{22} x_{2}
$$


where $\mathbf{w}_{21}$ and $\mathbf{w}_{22}$ are the transmit beamformers at $\mathrm{Tu}_{2}$ for $x_{1}$ and $x_{2}$, respectively, and they are designed to satisfy the power constraint as

$$
\mathbf{w}_{21}^{\dagger} \mathbf{w}_{21}+\mathbf{w}_{22}^{\dagger} \mathbf{w}_{22} \leq P_{2}
$$

where $P_{2}$ is the maximum transmit budget at $\mathrm{Tu}_{2}$ and $\mathbf{w}_{21}^{\dagger} \mathbf{w}_{21}=\beta P_{2}$. If $\mathrm{Tu}_{2}$ does not help $\mathrm{Tu}_{1}$ with probability $1-\alpha, \mathrm{Tu}_{2}$ does not allocate the power for $x_{1}$ such as $\beta=0$. Therefore, the received signals at $\mathrm{Ru}_{1}$ and $\mathrm{Ru}_{2}$ in the time slot $t_{2}$ are respectively given by

$y_{\mathrm{Ru}_{1}, 2}=\mathbf{h}_{21}^{\dagger} \mathbf{x}_{\mathrm{Tu}_{2}}+n_{\mathrm{Ru}_{1}, 2}=\mathbf{h}_{21}^{\dagger} \mathbf{w}_{21} x_{1}+\mathbf{h}_{21}^{\dagger} \mathbf{w}_{22} x_{2}+n_{\mathrm{Ru}_{1}, 2}$,

$y_{\mathrm{Ru}_{2}, 2}=\mathbf{h}_{2}^{\dagger} \mathbf{x}_{\mathrm{Tu}_{2}}+n_{\mathrm{Ru}_{2}, 2}=\mathbf{h}_{2}^{\dagger} \mathbf{w}_{22} x_{2}+\mathbf{h}_{2}^{\dagger} \mathbf{w}_{21} x_{1}+n_{\mathrm{Ru}_{2}, 2}$,

where $n_{\mathrm{Ru}_{1}, 2}$ and $n_{\mathrm{Ru}_{2}, 2}$ are the AWGN at $\mathrm{Ru}_{1}$ and $\mathrm{Ru}_{2}$ with $\mathcal{C N}\left(0, \sigma^{2}\right)$ in $t_{2}$, respectively. If $\mathrm{Ru}_{2}$ successfully decodes the data from $\mathrm{Tu}_{1}$ in the time slot $t_{1}, \mathrm{Ru}_{2}$ can subtract it from the received signal in $t_{2}$ by applying the successive interference cancellation (SIC). For this case, after applying $\mathrm{SIC}$, the received signal at $\mathrm{Ru}_{2}$ in $t_{2}$ can be rewritten as $y_{\mathrm{Ru}_{2}, 2}^{\mathrm{SIC}}=\mathbf{h}_{2}^{\dagger} \mathbf{w}_{22} x_{2}+n_{\mathrm{Ru}_{2}, 2}$. Otherwise, $\mathrm{Ru}_{2}$ has to decode $x_{2}$ by treating the signal related to $x_{1}$ as the noise.

In this paper, we only consider the case that $\mathrm{Ru}_{2}$ can always achieve its QoS requirement, $Q$, for given power budget, $P_{2}$, and hence, the $\mathrm{QoS}$ requirement at $\mathrm{Ru}_{2}$ is given in the range of $0 \leq Q \leq Q^{\max }$, where the maximum QoS requirement is $Q^{\max }=\frac{1}{2} \log _{2}\left(1+\frac{P_{2}\left\|\mathbf{h}_{2}\right\|^{2}}{\sigma^{2}}\right)$, which is achieved by the maximal-ratio transmission $(\mathrm{MRT})$ at $\mathrm{Tu}_{2}, \mathbf{w}_{2}^{\mathrm{mrt}}=\sqrt{P_{2}} \frac{\mathbf{h}_{2}}{\left\|\mathbf{h}_{2}\right\|}$ with maximum power $P_{2}$, and $\frac{1}{2}$ is from the fact that the transmission takes place in two time slots.

\section{USER COOPERATION BASED ON TRUSt DEGREE}

In this section, for given trust degree, we provide the cooperation strategy, which includes the transmission beamformer at $\mathrm{Tu}_{1}$ and the power allocation for cooperation at $\mathrm{Tu}_{2}$, to maximize the expected achievable rate at $\mathrm{Ru}_{1}$ while guaranteeing QoS requirement at $\mathrm{Ru}_{2}$. We derive the optimal transmission strategies for three cases: 1) SISO case $\left(N_{1}=N_{2}=1\right), 2$ ) MISO case $\left(N_{1} \geq 2, N_{2}=1\right)$, 3) SIMO case $\left(N_{1}=1\right.$, $\left.N_{2} \geq 2\right)$, and 4) MIMO case $\left(N_{1} \geq 2, N_{2} \geq 2\right)$. We first define the event of cooperation as $\mathcal{E}$, where $\mathcal{E}=1$ and $\mathcal{E}=0$ stand for the events that $\mathrm{Tu}_{2}$ helps and does not help the transmission, respectively. Thus, $\mathcal{E}$ is a Bernoulli random variable with $\operatorname{Pr}[\mathcal{E}=1]=\alpha$ and $\operatorname{Pr}[\mathcal{E}=0]=1-\alpha$. As a performance metric, for given trust degree $\alpha$, we use the expected achievable rate with respect to the possible cooperation events, defined as

$$
R_{\mathrm{Ru}_{1}}=\underset{\mathcal{E}}{\mathbb{E}}\left\{\tilde{R}_{\mathrm{Ru}_{1}}\right\}
$$

where $\tilde{R}_{\mathrm{Ru}_{1}}$ is an achievable rate at $\mathrm{Ru}_{1}$.

\section{A. SISO case $\left(N_{1}=N_{2}=1\right)$}

We first consider a simple SISO case that $\mathrm{Tu}_{1}$ and $\mathrm{Tu}_{2}$ have a single antenna $\left(N_{1}=N_{2}=1\right)$. We define the gains of all channels as

$$
g_{0}=\left|h_{0}\right|^{2}, g_{1}=\left|h_{1}\right|^{2}, g_{2}=\left|h_{2}\right|^{2}, g_{12}=\left|h_{12}\right|^{2}, g_{21}=\left|h_{21}\right|^{2} \text {. (9) }
$$

When the channel condition between $\mathrm{Tu}_{1}$ and $\mathrm{Tu}_{2}$ is worse than the direct channel from $\mathrm{Tu}_{1}$ to $\mathrm{Ru}_{1}$ (i.e. $g_{0} \leq g_{1}$ ), the cooperation of $\mathrm{Tu}_{2}$ cannot improve the achievable rate at $\mathrm{Ru}_{1}$ due to DF relaying constraint [22]. Thus, in this case, the achievable rate at $\mathrm{Ru}_{1}$ is achieved by the direct transmission from $\mathrm{Tu}_{1}$. Therefore, since $\mathrm{Tu}_{2}$ helps the transmission of $\mathrm{Tu}_{1}$ with probability $\alpha$, the expected achievable rate at $\mathrm{Ru}_{1}$ is given by

$$
R_{\mathrm{Ru}_{1}}(\beta)= \begin{cases}\bar{R}_{\mathrm{Ru}_{1}}(\beta), & \text { if } g_{0}>g_{1}, \\ \frac{1}{2} \log _{2}\left(1+\rho_{1} g_{1}\right), & \text { otherwise, }\end{cases}
$$

where $\bar{R}_{\mathrm{Ru}_{1}}(\beta)$ is given by

$$
\begin{aligned}
\bar{R}_{\mathrm{Ru}_{1}}(\beta)= & \frac{\alpha}{2} \min \left[\log _{2}\left(1+\rho_{1} g_{1}+\frac{\beta \rho_{2} g_{21}}{(1-\beta) \rho_{2} g_{21}+1}\right),\right. \\
& \left.\log _{2}\left(1+\rho_{1} g_{0}\right)\right]+\frac{1-\alpha}{2} \log _{2}\left(1+\rho_{1} g_{1}\right)
\end{aligned}
$$

where $\rho_{1}=\frac{P_{1}}{\sigma^{2}}$ and $\rho_{2}=\frac{P_{2}}{\sigma^{2}}$. From the observations of 10. and 111) in SISO case, we deduce that $R_{\mathrm{Ru}_{1}}$ is an increasing function of $\rho_{1}$. Therefore, as $P_{1}$ grows, the value of $R_{\mathrm{Ru}_{1}}$ is increasing, and the maximum transmit power $P_{1}$ is always optimal. The first term of (11) denotes the achievable rate at $\mathrm{Ru}_{1}$ when $\mathrm{Tu}_{2}$ helps transmission of $\mathrm{Tu}_{1}$ with probability $\alpha$, and it is bounded by the minimum of achievable rates at $\mathrm{Tu}_{2}$ and $\mathrm{Ru}_{1}$ due to the constraint of DF relaying [22]. The second term of 111 represents the achievable rate achieved by direct transmission from $\mathrm{Tu}_{1}$ to $\mathrm{Ru}_{1}$ when $\mathrm{Tu}_{2}$ does not help transmission of $\mathrm{Tu}_{1}$ with probability $(1-\alpha)$. In the SISO case, we can see that $\beta$ can be determined independently from $\alpha$. The power allocation $\beta$ has to be jointly determined with the transmit strategy at $\mathrm{Tu}_{1}$, which is related to $\alpha$. However, in SISO case, $\mathrm{Tu}_{1}$ does not design the transmit beamformer and hence, $\beta$ can be determined independently with $\alpha$ to maximize the rate achieved when $\mathrm{Tu}_{2}$ cooperates with $\mathrm{Tu}_{1}$.

We define the part related with $\beta$ in 111 as $Q_{\mathrm{Tu}_{1}}(\beta)$, given by

$$
\begin{aligned}
Q_{\mathrm{Tu}_{1}}(\beta)= & \frac{1}{2} \min \left[\log _{2}\left(1+\rho_{1} g_{1}+\frac{\beta \rho_{2} g_{21}}{(1-\beta) \rho_{2} g_{21}+1}\right),\right. \\
& \left.\log _{2}\left(1+\rho_{1} g_{0}\right)\right]
\end{aligned}
$$

According to $\beta, Q_{\mathrm{Tu}_{1}}(\beta)$ can be rewritten by

$$
Q_{\mathrm{Tu}_{1}}(\beta)= \begin{cases}\frac{1}{2} \log _{2}\left(1+\rho_{1} g_{0}\right), & \text { if } \beta_{0} \leq \beta \leq 1 \\ \frac{1}{2} \log _{2}\left(1+\rho_{1} g_{1}+\frac{\beta \rho_{2} g_{21}}{(1-\beta) \rho_{2} g_{21}+1}\right), & \text { otherwise }\end{cases}
$$

where $\beta_{0}=1-\frac{\rho_{2} g_{21}-\rho_{1}\left(g_{0}-g_{1}\right)}{\rho_{2} g_{21}\left\{1+\rho_{1}\left(g_{0}-g_{1}\right)\right\}}$.

For the case that $\mathrm{Tu}_{2}$ helps the transmission of $\mathrm{Tu}_{1} 1$, if the receiving rate at $\mathrm{Ru}_{2}$ in $t_{1}, R_{12}$, is greater than $Q_{\mathrm{Tu}_{1}}(\beta)$, i.e., $R_{12}=\frac{1}{2} \log _{2}\left(1+\rho_{1} g_{12}\right) \geq Q_{\mathrm{Tu}_{1}}(\beta), \mathrm{Ru}_{2}$ can decode $x_{1}$ in $t_{1}$ and hence, $\mathrm{Ru}_{2}$ can apply $\mathrm{SIC}$ to eliminate the effect of $x_{1}$

\footnotetext{
${ }^{1}$ Since the QoS requirement, $Q$, is always satisfied when $\mathrm{Tu}_{2}$ does not help the transmission of $\mathrm{Tu}_{1}$, we only consider the case that $\mathrm{Tu}_{2}$ helps the transmission of $\mathrm{Tu}_{1}$ to design the transmission strategy.
} 
in its received signal in $t_{2}$. Therefore, the achievable rate at $\mathrm{Ru}_{2}$ is given by

$$
R_{R u_{2}}(\beta)= \begin{cases}R_{\mathrm{Ru}}^{\mathrm{SIC}}(\beta), & \text { if } R_{12} \geq Q_{\mathrm{Tu}_{1}}(\beta), g_{0}>g_{1}, \\ R_{\mathrm{Ru}_{2}}^{\mathrm{NSIC}}(\beta), & \text { if } R_{12}<Q_{\mathrm{Tu}_{1}}(\beta), g_{0}>g_{1}, \\ \frac{1}{2} \log _{2}\left(1+\rho_{2} g_{2}\right), \text { otherwise, }\end{cases}
$$

where $R_{\mathrm{Ru}_{2}}^{\mathrm{SIC}}(\beta)$ and $R_{\mathrm{Ru}_{2}}^{\mathrm{NSIC}}(\beta)$ are given, respectively, by

$$
\begin{aligned}
& R_{\mathrm{Ru}_{2}}^{\mathrm{SIC}}(\beta)=\frac{1}{2} \log _{2}\left(1+(1-\beta) \rho_{2} g_{2}\right), \\
& R_{\mathrm{Ru}_{2}}^{\mathrm{NSIC}}(\beta)=\frac{1}{2} \log _{2}\left(1+\frac{(1-\beta) \rho_{2} g_{2}}{\beta \rho_{2} g_{2}+1}\right) .
\end{aligned}
$$

For the SISO case, the optimal power allocation of $\mathrm{Tu}_{2}$ that maximizes the expected achievable rate at $\mathrm{Ru}_{1}$ while guaranteeing QoS requirement at $\mathrm{Ru}_{2}$ is obtained by the following problem

$$
\begin{aligned}
\text { P1 : } \max _{0 \leq \beta \leq 1} R_{\mathrm{Ru}_{1}}(\beta) \\
\text { s.t. } R_{\mathrm{Ru}_{2}}(\beta) \geq Q,
\end{aligned}
$$

where $R_{\mathrm{Ru}_{1}}(\beta)$ is given in (10) and $Q \in\left[0, Q^{\max }\right]$ is QoS of $\mathrm{Ru}_{2}$, where $Q^{\max }=\frac{1}{2} \log _{2}\left(1+\rho_{2} g_{2}\right)$.

For given channel conditions and $\mathrm{QoS}$ at $\mathrm{Ru}_{2}$, we obtain the optimal power allocation of $\mathrm{Tu}_{2}$ for cooperative transmission in the following theorem.

Theorem 1: For given channels and QoS requirement, $Q$, the optimal power allocation of $\mathrm{Tu}_{2}$ for cooperative transmission that maximizes the expected achievable rate at $\mathrm{Ru}_{1}$ is obtained by

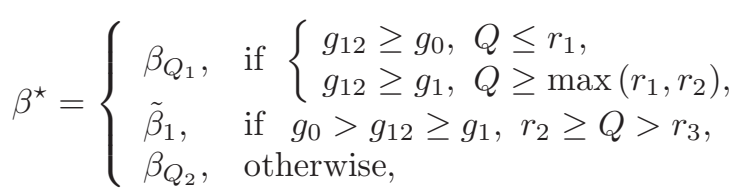

where $\beta_{Q_{1}}, \beta_{Q_{2}}$, and $\tilde{\beta}_{1}$ are given, respectively, by

$$
\begin{aligned}
& \beta_{Q_{1}}=1-\frac{4^{Q}-1}{\rho_{2} g_{2}}, \\
& \beta_{Q_{2}}=\left(1-\frac{4^{Q}-1}{\rho_{2} g_{2}}\right) 4^{-Q}, \\
& \tilde{\beta}_{1}=1-\frac{\rho_{2} g_{21}-\rho_{1}\left(g_{12}-g_{1}\right)}{\rho_{2} g_{21}\left\{1+\rho_{1}\left(g_{12}-g_{1}\right)\right\}},
\end{aligned}
$$

and $r_{1}, r_{2}$ and $r_{3}$ are given by

$$
\begin{aligned}
& r_{1}=\left[\frac{1}{2} \log _{2}\left(1+\left(1-\beta_{0}\right) \rho_{2} g_{2}\right)\right]^{+}, \\
& r_{2}=\left[\frac{1}{2} \log _{2}\left(1+\left(1-\tilde{\beta}_{1}\right) \rho_{2} g_{2}\right)\right]^{+}, \\
& r_{3}=\frac{1}{2} \log _{2}\left(\frac{1+\rho_{2} g_{2}}{1+\rho_{2} g_{2} \tilde{\beta}_{1}}\right) .
\end{aligned}
$$

Proof: The proof is presented in Appendix A

Remark 1: From Theorem 1, we observe that the optimal $\beta$ that maximizes the expected achievable rate at $\mathrm{Ru}_{1}$ while guaranteeing QoS of $\mathrm{Ru}_{2}$ is mainly determined by channel quality from $\mathrm{Tu}_{1}$ to $\mathrm{Ru}_{2}, g_{12}$ and $\mathrm{QoS}$ of $\mathrm{Ru}_{2}, Q$. Since $\mathrm{Tu}_{2}$ can assign more power for cooperation when $\mathrm{Ru}_{2}$ can apply SIC than that when $\mathrm{Ru}_{2}$ cannot apply SIC, the optimal power allocation is mainly determined by the parameters that decide whether SIC is applicable at $\mathrm{Ru}_{2}, g_{12}$ and $Q$.
For the case that the channel quality from $\mathrm{Tu}_{1}$ to $\mathrm{Ru}_{2}$ is good such as $g_{12} \geq g_{0}$ and $g_{12} \geq g_{1}$, since we have $r_{1} \geq r_{2}$ from $\beta_{0} \leq \tilde{\beta}_{1}$, the optimal power allocation for cooperation is given by $\beta^{\star}=\beta_{Q_{1}}$ for all $Q$. In this case, for all $Q, \mathrm{Ru}_{2}$ can apply SIC due to good channel quality between $\mathrm{Tu}_{1}$ and $\mathrm{Ru}_{2}$. Contrarily, when the channel quality from $\mathrm{Tu}_{1}$ to $\mathrm{Ru}_{2}$ is poor such as $g_{0}>g_{12}$ and $g_{1}>g_{12}, \mathrm{Ru}_{2}$ cannot apply SIC for all $Q$ and hence, the $\mathrm{Tu}_{2}$ allocates the minimum power for cooperation as $\beta^{\star}=\beta_{Q_{2}}$.

On the other hand, for the moderate quality of $g_{12}$ as $g_{0}>$ $g_{12} \geq g_{1}$, the power allocation is also determined according to QoS requirement, $Q$. In this case, since $\mathrm{Ru}_{2}$ cannot always apply SIC due to $g_{12}$ and $Q, \beta$ has to be controlled to apply SIC. First, for the case that QoS requirement is small such as $Q \leq r_{3}$, since $\mathrm{Tu}_{2}$ can allocate large power for cooperation without SIC as $\beta^{\star}=\beta_{Q_{2}}$, which is a decreasing function with $Q$, SIC by reducing $\beta$ is not beneficial. For moderate QoS as $r_{2} \geq Q>r_{3}, \mathrm{Tu}_{2}$ controls $\beta$ as $\beta^{\star}=\tilde{\beta}_{1}$, which is a constant for given channels, to apply SIC. In the moderate $Q$, the power for cooperation is not decreased even if $Q$ increases. However, when QoS requirement is high as $Q \geq r_{2}$, the power allocation for cooperation is decreased according to $Q$ as $\beta^{\star}=\beta_{Q_{1}}$ to guarantee high QoS by applying SIC.

\section{B. $\operatorname{MISO}$ case $\left(N_{1} \geq 2\right.$ and $\left.N_{2}=1\right)$}

In this subsection, we consider the MISO case, where $\mathrm{Tu}_{1}$ equips with multiple antennas (i.e., $N_{1} \geq 2$ ) but $\mathrm{Tu}_{2}$ has a single antenna (i.e., $N_{2}=1$ ). Hence, in the MISO case, we jointly design the transmit beamformer at $\mathrm{Tu}_{1}, \mathrm{w}_{1}$, and the power allocation for cooperation at $\mathrm{Tu}_{2}, \beta$, to maximize the expected achievable rate at $\mathrm{Ru}_{1}$ while guaranteeing the QoS requirement at $\mathrm{Ru}_{2}$. For the MISO case, the expected achievable rate at $\mathrm{Ru}_{1}$ is given by

$$
R_{\mathrm{Ru}_{1}}\left(\mathbf{w}_{1}, \beta\right)= \begin{cases}\bar{R}_{\mathrm{Ru}_{1}}\left(\mathbf{w}_{1}, \beta\right), & \text { if } \tilde{g}_{0}>\tilde{g}_{1}, \\ \frac{1}{2} \log _{2}\left(1+\rho_{1} \tilde{g}_{1}\right), & \text { otherwise }\end{cases}
$$

where $\tilde{g}_{0}=\left\|\mathbf{h}_{0}\right\|^{2}, \tilde{g}_{1}=\left\|\mathbf{h}_{1}\right\|^{2}$ and $\bar{R}_{\mathrm{Ru}_{1}}\left(\mathbf{w}_{1}, \beta\right)$ is given by

$$
\begin{array}{r}
\bar{R}_{\mathrm{Ru}_{1}}\left(\mathbf{w}_{1}, \beta\right)=\frac{\alpha}{2} \min \left[\log _{2}\left(1+\rho_{1}\left|\mathbf{h}_{1}^{\dagger} \mathbf{w}_{1}\right|^{2}+\frac{\beta \rho_{2} g_{21}}{(1-\beta) \rho_{2} g_{21}+1}\right),\right. \\
\left.\log _{2}\left(1+\rho_{1}\left|\mathbf{h}_{0}^{\dagger} \mathbf{w}_{1}\right|^{2}\right)\right]+\frac{1-\alpha}{2} \log _{2}\left(1+\rho_{1}\left|\mathbf{h}_{1}^{\dagger} \mathbf{w}_{1}\right|^{2}\right) .
\end{array}
$$

The part related with both $\mathbf{w}_{1}$ and $\beta$ in 23 is defined by

$$
\begin{aligned}
Q_{\mathrm{Tu}_{1}}\left(\mathbf{w}_{1}, \beta\right)= & \frac{1}{2} \min \left[\log _{2}\left(1+\rho_{1}\left|\mathbf{h}_{1}^{\dagger} \mathbf{w}_{1}\right|^{2}+\frac{\beta \rho_{2} g_{21}}{(1-\beta) \rho_{2} g_{21}+1}\right),\right. \\
& \left.\log _{2}\left(1+\rho_{1}\left|\mathbf{h}_{0}^{\dagger} \mathbf{w}_{1}\right|^{2}\right)\right] .
\end{aligned}
$$

Similar to SISO case, the expected achievable rate at $\mathrm{Ru}_{1}$ is an increasing function of $P_{1}$ and hence, the maximum transmit power $P_{1}$ is always optimal for MISO case.

For the case that $\mathrm{Tu}_{2}$ helps the transmission of $\mathrm{Tu}_{1}$, if the receiving rate at $\mathrm{Ru}_{2}$ in $t_{1}, R_{12}\left(\mathbf{w}_{1}\right)$, is greater than $Q_{\mathrm{Tu}_{1}}\left(\mathbf{w}_{1}, \beta\right)$, i.e., $R_{12}\left(\mathbf{w}_{1}\right)=\frac{1}{2} \log _{2}\left(1+\rho_{1}\left|\mathbf{h}_{12}^{\dagger} \mathbf{w}_{1}\right|^{2}\right) \geq$ $Q_{\mathrm{Tu}_{1}}\left(\mathbf{w}_{1}, \beta\right), \mathrm{Ru}_{2}$ can decode $x_{1}$ in $t_{1}$ and hence, $\mathrm{Ru}_{2}$ can 
apply SIC to eliminate the effect of $x_{1}$ in its received signal in $t_{2}$. Therefore, the achievable rate at $\mathrm{Ru}_{2}$ is given by

$R_{R u_{2}}(\beta)=\left\{\begin{array}{l}R_{\mathrm{Ru}}^{\mathrm{SIC}}(\beta), \text { if } R_{12}\left(\mathbf{w}_{1}\right) \geq Q_{\mathrm{Tu}_{1}}\left(\mathbf{w}_{1}, \beta\right), \tilde{g_{0}}>\tilde{g_{1}}, \\ R_{\mathrm{Ru}_{2}}^{\mathrm{NSIC}}(\beta), \text { if } R_{12}\left(\mathbf{w}_{1}\right)<Q_{\mathrm{Tu}_{1}}\left(\mathbf{w}_{1}, \beta\right), \tilde{g_{0}}>\tilde{g_{1}}, \\ \frac{1}{2} \log _{2}\left(1+\rho_{2} g_{2}\right), \text { otherwise, }\end{array}\right.$

where $R_{\mathrm{Ru}}^{\mathrm{SIC}}(\beta)$ and $R_{\mathrm{Ru} 2}^{\mathrm{NSIC}}(\beta)$ are given in (15).

For the MISO case, the optimal beamformer at $\mathrm{Tu}_{1}, \mathbf{w}_{1}$, and the power allocation for cooperation at $\mathrm{Tu}_{2}, \beta$, that maximize the expected achievable rate at $\mathrm{Ru}_{1}$ while guaranteeing $\mathrm{QoS}$ requirement at $\mathrm{Ru}_{2}$ are obtained by solving the following joint optimization problem

$$
\begin{array}{r}
\text { P2: } \max _{\mathbf{w}_{1}, 0 \leq \beta \leq 1} R_{\mathrm{Ru}_{1}}\left(\mathbf{w}_{1}, \beta\right) \\
\text { s.t. } R_{\mathrm{Ru}_{2}}(\beta) \geq Q, \quad \mathbf{w}_{1}^{\dagger} \mathbf{w}_{1} \leq 1 .
\end{array}
$$

We define the constant values for given channels, $v_{1}, v_{2}$ and $\phi_{1}$ as

$$
v_{1} \triangleq\left\|\boldsymbol{\Pi}_{\mathbf{h}_{0}} \mathbf{h}_{1}\right\|^{2}, v_{2} \triangleq\left\|\boldsymbol{\Pi}_{\mathbf{h}_{0}}^{\perp} \mathbf{h}_{1}\right\|^{2}, \phi_{1} \triangleq \rho_{1}\left(1+\frac{1}{\rho_{2} g_{21}}\right) .
$$

Then, the optimal structure of beamformer $\mathbf{w}_{1}$ can be obtained by the following lemma.

Lemma 1: [15, Lemma 1] The optimal beamformer at $\mathrm{Tu}_{1}$ that maximizes the expected achievable rate at $\mathrm{Ru}_{1}$ can be represented by

$$
\mathbf{w}_{1}^{\text {opt }}=\sqrt{\eta} \mathbf{w}_{0}+\sqrt{1-\eta} \mathbf{w}_{0}^{\perp},
$$

where $\mathbf{w}_{0}=\frac{\boldsymbol{\Pi}_{\mathbf{h}_{0}} \mathbf{h}_{1}}{\left\|\boldsymbol{\Pi}_{\mathbf{h}_{0}} \mathbf{h}_{1}\right\|}, \mathbf{w}_{0}^{\perp}=\frac{\boldsymbol{\Pi}_{\mathbf{h}_{0}}^{\perp} \mathbf{h}_{1}}{\left\|\boldsymbol{\Pi}_{\mathbf{h}_{0}}^{\perp} \mathbf{h}_{1}\right\|}$ and $\eta$ is a constant in the range of $\frac{v_{1}}{v_{1}+v_{2}} \leq \eta \leq 1$.

Proof: The proof of Lemma 1 can be referred to [15].

In Lemma 1, it is difficult to present $\eta$ that maximizes $\bar{R}_{\mathrm{Ru}_{1}}\left(\mathbf{w}_{1}, \beta\right)$ in closed form and hence, the optimal $\eta$ should be found by exhaustive search. However, from the numerically obtained beamformer $\mathbf{w}_{1}$, we cannot get the insight on the effect of trust degree on $\bar{R}_{\mathrm{Ru}_{1}}\left(\mathbf{w}_{1}, \beta\right)$. Hence, in order to obtain $\mathbf{w}_{1}$ in closed form, the approximated expected achievable rate at $\mathrm{Ru}_{1}$ can be obtained by high signal-to-noise ratio (SNR) approximation 2 , as

$$
\begin{gathered}
\tilde{R}_{\mathrm{Ru}_{1}}\left(\mathbf{w}_{1}, \beta\right) \approx \frac{\alpha}{2} \min \left[\log _{2}\left(\rho_{1}\left|\mathbf{h}_{1}^{\dagger} \mathbf{w}_{1}\right|^{2}+\frac{\beta \rho_{2} g_{21}}{(1-\beta) \rho_{2} g_{21}+1}\right),\right. \\
\left.\log _{2}\left(\rho_{1}\left|\mathbf{h}_{0}^{\dagger} \mathbf{w}_{1}\right|^{2}\right)\right]+\frac{1-\alpha}{2} \log _{2}\left(\rho_{1}\left|\mathbf{h}_{1}^{\dagger} \mathbf{w}_{1}\right|^{2}\right) .
\end{gathered}
$$

For given $\beta$, the transmit beamformer that maximizes the approximated expected achievable rate at $\mathrm{Ru}_{1}$ can be obtained by the following theorem.

Theorem 2: For given trust degree $\alpha$ and the power allocation $\beta$, the transmit beamformer of $\mathrm{Tu}_{1}$ that maximizes $\tilde{R}_{\mathrm{Ru}_{1}}\left(\mathbf{w}_{1}\right)$ is obtained by

$$
\mathbf{w}_{1}^{\star}=\sqrt{\eta^{\star}} \mathbf{w}_{0}+\sqrt{1-\eta^{\star}} \mathbf{w}_{0}^{\perp},
$$

where $\eta^{\star}$ is given by 30 .

\footnotetext{
${ }^{2}$ In order to obtain the closed-form beamformer, we adopt the high SNR approximation. However, it does not mean that we assume the high SNR configuration in our system model.
}

Proof: The proof is presented in Appendix B

Remark 2: From Theorem 2, we can see that the direction of beamformer at $\mathrm{Tu}_{1}, \eta$, is affected by both the trust degree, $\alpha$ and the power allocation for cooperation, $\beta$. First, when the power allocation for cooperation is very small such as $\beta<\beta$, the expected achievable rate enhancement from the cooperation with $\mathrm{Tu}_{2}$ is small even if $\alpha$ is large. Hence, in this case, the beamformer at $\mathrm{Tu}_{1}$ is designed to maximize the direct link from $\mathrm{Tu}_{1}$ to $\mathrm{Ru}_{1}$ regardless of $\alpha$. On the other hand, for high $\beta$ as $\beta>\bar{\beta}$, since the expected achievable rate enhancement is large enough due to high $\beta$, the direction of beamformer mainly depends on the trust degree, $\alpha$. Hence, when $\mathrm{Tu}_{2}$ helps the transmission of $\mathrm{Tu}_{1}$ with high probability, equivalently $\alpha$ is high, the direction of beamformer is steered toward $\mathbf{h}_{0}$ based on $\eta_{2}$, which is an increasing function with $\alpha$ to fully exploit the cooperation of $\mathrm{Tu}_{2}$. Otherwise, the direction of beamformer has to be properly steered based both $\alpha$ and $\beta$. When $\alpha$ is relatively high compared to $\beta$ such as $\eta_{2}>\eta_{3}(\beta)$, the direction of beamformer tends to be steered toward $\mathbf{h}_{0}$ rather than $\mathbf{h}_{1}$ and vice versa.

In Theorem 2, we show that beamformer $\mathbf{w}_{1}$ can be represented according to $\beta$. Thus, the joint optimization problem of $\mathbf{w}_{1}$ and $\beta$ can be simplified by the optimization problem of a single parameter $\beta$, as

$$
\begin{aligned}
\mathbf{P 2}-1: & \max _{0 \leq \beta \leq 1} R_{\mathrm{Ru}_{1}}(\beta) \\
& \text { s.t. } R_{\mathrm{Ru}_{2}}(\beta) \geq Q .
\end{aligned}
$$

For the general case, it is hard to directly obtain the optimal $\beta^{\star}$ from $\mathbf{P 2}-\mathbf{1}$ because $\mathbf{P 2}-\mathbf{1}$ is non-convex with respect to $\beta$. Thus, in the following corollaries, we obtain the optimal power allocation for some special cases. In the following corollaries, we assume $\rho_{1}=\rho_{2}$ for simplicity.

Corollary 1: When the channel from $\mathrm{Tu}_{1}$ to $\mathrm{Tu}_{2}$ is very strong such as $\tilde{g}_{0} \geq \overline{g_{0}} \triangleq \frac{\tilde{g}_{1}\left(\tilde{g}_{1}+g_{21}\right)}{v_{1}}$, the optimal power allocation for cooperation that maximizes the approximated achievable rate at $\mathrm{Ru}_{1}$ can be represented by

$$
\beta^{\star}= \begin{cases}\min \left\{\tilde{\beta}_{2}, \beta_{Q_{1}}\right\}, & \text { if } v_{3} \geq \tilde{g}_{1}^{2}, \\ \beta_{Q_{2}}, & \text { otherwise, }\end{cases}
$$

where $\tilde{\beta}_{2}$ and $v_{3}$ are given by

$$
\tilde{\beta}_{2}=\frac{\left(v_{3}-\tilde{g}_{1}^{2}\right) \phi_{1}}{\tilde{g}_{1}+\left(v_{3}-\tilde{g}_{1}^{2}\right) \rho_{1}}, \quad v_{3}=\left|\mathbf{h}_{12}^{\dagger} \mathbf{h}_{1}\right|^{2},
$$

and $\beta_{Q_{1}}$ and $\beta_{Q_{2}}$ are given in (18) and (19), respectively.

Proof: For $\tilde{g}_{0} \geq \overline{g_{0}}$, since we have $\underline{\beta} \geq 1$, for all feasible $\beta$ in $0 \leq \beta \leq 1$, the optimal beamformer is given by $\mathbf{w}_{1}^{\star}\left(\eta_{1}\right)=$ $\sqrt{\eta_{1}} \mathbf{w}_{0}+\sqrt{1-\eta_{1}} \mathbf{w}_{0}^{\perp}=\frac{\mathbf{h}_{1}}{\sqrt{\tilde{g}_{1}}}$ and the approximated achievable rate at $\mathrm{Ru}_{1}$ is represented by $B$. 3 with $\eta_{1}$. For this case, the condition to apply SIC at $\mathrm{Ru}_{2}$ is

$$
\begin{aligned}
R_{12}\left(\mathbf{w}_{1}^{\star}\right) & =\frac{1}{2} \log _{2}\left(1+\frac{\rho_{1} v_{3}}{\tilde{g}_{1}}\right) \\
& \geq \frac{1}{2} \log _{2}\left(1+\rho_{1}\left(\tilde{g}_{1}+m_{1}(\beta)\right)\right) \\
& =Q_{\mathrm{Tu}_{1}}\left(\mathbf{w}_{1}^{\star}, \beta\right) \Rightarrow \beta \leq \tilde{\beta}_{2} .
\end{aligned}
$$

If $v_{3}<\tilde{g}_{1}^{2}, \mathrm{Ru}_{2}$ cannot apply SIC for all $\beta$ and hence, for $v_{3} \geq$ $\tilde{g}_{1}^{2}$, the condition to apply SIC at $\mathrm{Ru}_{2}$ while guaranteeing $\mathrm{QoS}$ 


$$
\eta^{\star}= \begin{cases}\eta_{1}=\frac{v_{1}}{v_{1}+v_{2}}, & \text { if } \beta<\underline{\beta}=\frac{\left\{v_{1} \tilde{g}_{0}-\left(v_{1}+v_{2}\right)^{2}\right\} \phi_{1}}{v_{1}+v_{2}+\left\{v_{1} \tilde{g}_{0}-\left(v_{1}+v_{2}\right)^{2}\right\} \rho_{1}}, \tilde{g}_{0}>\frac{\left(v_{1}+v_{2}\right)^{2}}{v_{1}}, \\
\eta_{2}=\frac{v_{1}+2 v_{2} \alpha+\sqrt{v_{1}^{2}+4 v_{1} v_{2} \alpha(1-\alpha)}}{2\left(v_{1}+v_{2}\right)}, & \text { if }\left\{\begin{array}{l}
\beta>\bar{\beta}=\frac{\left(\tilde{g}_{0}-v_{1}\right) \phi_{1}}{1+\left(\tilde{g}_{0}-v_{1}\right) \rho_{1}}, \tilde{g}_{0} \geq v_{1}, \\
\tilde{g}_{0}<v_{1},
\end{array}\right. \\
\min \left\{\eta_{2}, \eta_{3}(\beta)\right\}, & \text { otherwise, }\end{cases}
$$

where

$$
\begin{aligned}
& \eta_{3}(\beta)=\frac{v_{2}\left(v_{1}+v_{2}+\tilde{g}_{0}\right)+m_{1}(\beta)\left(\tilde{g}_{0}-\left(v_{1}+v_{2}\right)\right)+2 \sqrt{v_{1} v_{2}\left\{v_{2} \tilde{g}_{0}+m_{1}(\beta)\left(\tilde{g}_{0}-\left(v_{1}+v_{2}\right)-m_{1}(\beta)\right)\right\}}}{\left(\tilde{g}_{0}-v_{1}\right)^{2}+v_{2}\left(2 v_{1}+v_{2}+2 \tilde{g}_{0}\right)}, \\
& m_{1}(\beta)=\frac{\beta}{\phi_{1}-\beta \rho_{1}} .
\end{aligned}
$$

requirement is obtained by $\beta \leq \min \left\{\tilde{\beta}_{2}, \beta_{Q_{1}}\right\}$. Since B.4 is an increasing function of $\beta$, the optimal power allocation is obtained by $\beta^{\star}=\min \left\{\tilde{\beta}_{2}, \beta_{Q_{1}}\right\}$.

Otherwise, $\mathrm{Ru}_{2}$ cannot apply SIC for all $\beta$ and hence, the power allocation to guarantee QoS without SIC is obtained by $\beta^{\star}=\beta_{Q_{2}}$.

Corollary 2: When the channel from $\mathrm{Tu}_{1}$ to $\mathrm{Tu}_{2}$ is very weak such as $\tilde{g}_{0}<v_{1}$, the optimal power allocation for cooperation that maximizes the approximated achievable rate at $\mathrm{Ru}_{1}$ can be represented by

$$
\beta^{\star}= \begin{cases}\beta_{Q_{1}}, & \text { if } v_{4} \geq v_{5}, \\ \beta_{Q_{2}}, & \text { otherwise, }\end{cases}
$$

where $v_{4}$ and $v_{5}$ are given by $v_{4}=\left|\mathbf{h}_{12}^{\dagger} \mathbf{w}_{1}^{\star}\left(\eta_{2}\right)\right|^{2}$ and $v_{5}=$ $\left|\mathbf{h}_{0}^{\dagger} \mathbf{w}_{1}^{\star}\left(\eta_{2}\right)\right|^{2}$.

Proof: If $\tilde{g}_{0}<v_{1}$, for all feasible $\beta$ in $0 \leq \beta \leq 1$, the optimal beamformer is given by $\mathbf{w}_{1}^{\star}\left(\eta_{2}\right)=\sqrt{\eta_{2}} \mathbf{w}_{0}+$ $\sqrt{1-\eta_{2}} \mathbf{w}_{0}^{\perp}$. Hence, the remaining part of the proof of Corollary 2 can be obtained in the similar way to the proof of Corollary 1.

From Lemma 11 Theorem 2 and high SNR approximation, the joint optimization problem to design beamformer at $\mathrm{Tu}_{1}$, $\mathbf{w}_{1}$, and the power allocation at $\mathrm{Tu}_{2}, \beta$, is simplified into the optimization problem with a single parameter $\beta$. For some special case, the optimal $\beta$ can be obtained in a closed form. For general case, the optimal power allocation for cooperation can be obtained by one dimensional search from 0 to 1 , which is much simpler than solving the joint optimization problem.

\section{C. $\operatorname{SIMO}$ case $\left(N_{1}=1\right.$ and $\left.N_{2} \geq 2\right)$}

In this subsection, we consider the SIMO case, where $\mathrm{Tu}_{1}$ equips with a single antenna (i.e., $N_{1}=1$ ) but $\mathrm{Tu}_{2}$ is equipped with $N_{2} \geq 2$ antennas and hence, the SIMO channel is formed between $\mathrm{Tu}_{1}$ and $\mathrm{Tu}_{2}$. For the SIMO case, we jointly design the transmit beamformers at $\mathrm{Tu}_{2}, \mathbf{w}_{21}$ and $\mathbf{w}_{22}$, to maximize the expected achievable rate at $\mathrm{Ru}_{1}$. For this case, the expected achievable rate at $\mathrm{Ru}_{1}$ in SIMO case is given by

$$
R_{\mathrm{Ru}_{1}}\left(\mathbf{w}_{21}, \mathbf{w}_{22}\right)= \begin{cases}\bar{R}_{\mathrm{Ru}_{1}}\left(\mathbf{w}_{21}, \mathbf{w}_{22}\right), & \text { if } \tilde{g}_{0}>g_{1}, \\ \frac{1}{2} \log _{2}\left(1+\rho_{1} g_{1}\right), & \text { otherwise }\end{cases}
$$

where $\tilde{g}_{0}>g_{1}$ means the channel condition between $\mathrm{Tu}_{1}$ and $\mathrm{Tu}_{2}$ is better than the direct channel from $\mathrm{Tu}_{1}$ to $\mathrm{Ru}_{1}$, thus the cooperation of $\mathrm{Tu}_{2}$ can improve the achievable rate at $\mathrm{Ru}_{1}$, and $\bar{R}_{\mathrm{Ru}_{1}}\left(\mathbf{w}_{21}, \mathbf{w}_{22}\right)$ is given by

$$
\begin{aligned}
\bar{R}_{\mathrm{Ru}_{1}}\left(\mathbf{w}_{21}, \mathbf{w}_{22}\right)= & \frac{\alpha}{2} \min \left[\log _{2}\left(1+\rho_{1} g_{1}+\frac{\left|\mathbf{h}_{21}^{\dagger} \mathbf{w}_{21}\right|^{2}}{\left|\mathbf{h}_{21}^{\dagger} \mathbf{w}_{22}\right|^{2}+\sigma^{2}}\right),\right. \\
& \left.\log _{2}\left(1+\rho_{1} \tilde{g}_{0}\right)\right]+\frac{1-\alpha}{2} \log _{2}\left(1+\rho_{1} g_{1}\right) .
\end{aligned}
$$

We define the first term in 39 as

$$
\begin{aligned}
Q_{\mathrm{Tu}_{1}}\left(\mathbf{w}_{21}, \mathbf{w}_{22}\right)= & \frac{1}{2} \min \left[\log _{2}\left(1+\rho_{1} g_{1}+\frac{\left|\mathbf{h}_{21}^{\dagger} \mathbf{w}_{21}\right|^{2}}{\left|\mathbf{h}_{21}^{\dagger} \mathbf{w}_{22}\right|^{2}+\sigma^{2}}\right),\right. \\
& \left.\log _{2}\left(1+\rho_{1} \tilde{g}_{0}\right)\right] .
\end{aligned}
$$

For the case that $\mathrm{Tu}_{2}$ helps the transmission of $\mathrm{Tu}_{1}$, if the rate achieved at $\mathrm{Ru}_{2}$ in $t_{1}, R_{12}$, is greater than $Q_{\mathrm{Tu}_{1}}\left(\mathbf{w}_{21}, \mathbf{w}_{22}\right)$, i.e., $R_{12}=\frac{1}{2} \log _{2}\left(1+\rho_{1} g_{12}\right) \geq$ $Q_{\mathrm{Tu}_{1}}\left(\mathbf{w}_{21}, \mathbf{w}_{22}\right), \mathrm{Ru}_{2}$ can decode $x_{1}$ in $t_{1}$ and thus, $\mathrm{Ru}_{2}$ can employ SIC to eliminate the effect of $x_{1}$ in its received signal in $t_{2}$. Therefore, the achievable rate at $\mathrm{Ru}_{2}$ is given by

$$
R_{\mathrm{Ru}_{2}}\left(\mathbf{w}_{21}, \mathbf{w}_{22}\right)=\left\{\begin{array}{l}
R_{\mathrm{Ru}_{2}}^{\mathrm{SIC}}\left(\mathbf{w}_{22}\right), \text { if }\left\{\begin{array}{l}
R_{12} \geq Q_{\mathrm{Tu}_{1}}\left(\mathbf{w}_{21}, \mathbf{w}_{22}\right) \\
\tilde{g}_{0}>g_{1}
\end{array}\right. \\
R_{\mathrm{Ru}_{2}}^{\mathrm{NSIC}}\left(\mathbf{w}_{21}, \mathbf{w}_{22}\right), \text { if }\left\{\begin{array}{l}
R_{12}<Q_{\mathrm{Tu}_{1}}\left(\mathbf{w}_{21}, \mathbf{w}_{22}\right) \\
\tilde{g}_{0}>g_{1}
\end{array}\right. \\
\frac{1}{2} \log _{2}\left(1+\rho_{2} \tilde{g}_{2}\right), \text { otherwise, }
\end{array}\right.
$$

where $R_{\mathrm{Ru}_{2}}^{\mathrm{SIC}}\left(\mathbf{w}_{22}\right)$ and $R_{\mathrm{Ru}_{2}}^{\mathrm{NSIC}}\left(\mathbf{w}_{21}, \mathbf{w}_{22}\right)$ are respectively given by

$$
\begin{aligned}
& R_{\mathrm{Ru}_{2}}^{\mathrm{SIC}}\left(\mathbf{w}_{22}\right)=\frac{1}{2} \log _{2}\left(1+\frac{\left|\mathbf{h}_{2}^{\dagger} \mathbf{w}_{22}\right|^{2}}{\sigma^{2}}\right), \\
& R_{\mathrm{Ru}_{2}}^{\mathrm{NSIC}}\left(\mathbf{w}_{21}, \mathbf{w}_{22}\right)=\frac{1}{2} \log _{2}\left(1+\frac{\left|\mathbf{h}_{2}^{\dagger} \mathbf{w}_{22}\right|^{2}}{\left|\mathbf{h}_{2}^{\dagger} \mathbf{w}_{21}\right|^{2}+\sigma^{2}}\right) .
\end{aligned}
$$

For the SIMO case, to maximize the expected achievable rate at $\mathrm{Ru}_{1}$ while guaranteeing QoS of $\mathrm{Ru}_{2}$, the beamformers at $\mathrm{Tu}_{2}, \mathbf{w}_{21}$ and $\mathbf{w}_{22}$, are jointly optimized by the following problem

$$
\text { P3: } \begin{aligned}
\max _{\mathbf{w}_{21}, \mathbf{w}_{22}} & R_{\mathrm{Ru}_{1}}\left(\mathbf{w}_{21}, \mathbf{w}_{22}\right) \\
\text { s.t. } & R_{\mathrm{Ru}_{2}}\left(\mathbf{w}_{21}, \mathbf{w}_{22}\right) \geq Q, \\
& \mathbf{w}_{21}^{\dagger} \mathbf{w}_{21}+\mathbf{w}_{22}^{\dagger} \mathbf{w}_{22} \leq P_{2},
\end{aligned}
$$


where the power allocation at $\mathrm{Tu}_{2}$ is embedded in the beamformer design.

By considering the condition whether $\mathrm{Ru}_{2}$ applies SIC, P3 in (43) can be divided by two subproblems. For the case that $\mathrm{Ru}_{2}$ applies $\mathrm{SIC}$, the optimization problem $\mathbf{P} \mathbf{3} \mathbf{1}$ is given by

$$
\begin{aligned}
& \text { P3 - 1 } \max _{\mathbf{w}_{21}, \mathbf{w}_{22}} R_{\mathrm{Ru}_{1}}\left(\mathbf{w}_{21}, \mathbf{w}_{22}\right) \\
& \text { s.t. } \frac{1}{2} \log _{2}\left(1+\rho_{1} g_{12}\right) \geq Q_{\mathrm{Tu}_{1}}\left(\mathbf{w}_{21}, \mathbf{w}_{22}\right) \text {, } \\
& \frac{1}{2} \log _{2}\left(1+\frac{\left|\mathbf{h}_{2}^{\dagger} \mathbf{w}_{22}\right|^{2}}{\sigma^{2}}\right) \geq Q, \\
& \mathbf{w}_{21}^{\dagger} \mathbf{w}_{21}+\mathbf{w}_{22}^{\dagger} \mathbf{w}_{22} \leq P_{2},
\end{aligned}
$$

where constraint 44b is the condition that $\mathrm{Ru}_{2}$ decodes the data from $\mathrm{Tu}_{1}$ in $t_{1}$ and applies SIC to cancel it in $t_{2}$. Similarly, the case that $\mathrm{Ru}_{2}$ does not apply SIC, $\mathbf{P 3}-\mathbf{2}$, is given by

$$
\begin{aligned}
\mathbf{P 3}-\mathbf{2} \max _{\mathbf{w}_{21}, \mathbf{w}_{22}} & R_{\mathrm{Ru}_{1}}\left(\mathbf{w}_{21}, \mathbf{w}_{22}\right) \\
\text { s.t. } & \frac{1}{2} \log _{2}\left(1+\rho_{1} g_{12}\right)<Q_{\mathrm{Tu}_{1}}\left(\mathbf{w}_{21}, \mathbf{w}_{22}\right), \\
& \frac{1}{2} \log _{2}\left(1+\frac{\left|\mathbf{h}_{2}^{\dagger} \mathbf{w}_{22}\right|^{2}}{\left|\mathbf{h}_{2}^{\dagger} \mathbf{w}_{21}\right|^{2}+\sigma^{2}}\right) \geq Q, \\
& \mathbf{w}_{21}^{\dagger} \mathbf{w}_{21}+\mathbf{w}_{22}^{\dagger} \mathbf{w}_{22} \leq P_{2} .
\end{aligned}
$$

For the relaying case, i.e., $\tilde{g}_{0}>g_{1}$, from (39), $R_{\mathrm{Ru}_{1}}\left(\mathbf{w}_{21}, \mathbf{w}_{22}\right)$ can be presented by two different forms according to $\mathbf{w}_{21}$ and $\mathbf{w}_{22}$ due to DF relaying constraint. Therefore, both P3 $\mathbf{- 1}$ and P3 $\mathbf{- 2}$ can be further divided into two subproblems with respect to $R_{\mathrm{Ru}_{1}}\left(\mathbf{w}_{21}, \mathbf{w}_{22}\right)$. Hence, we can obtain the solution of P3 in (43) by choosing the best solution from the solutions of four subproblems. Since all subproblems can be solved in a similar way, here we focus on one of the four subproblems. In the following, we consider the subproblem $\mathbf{P} \mathbf{3}-\mathbf{2 1}$, where $\mathrm{Ru}_{2}$ does not apply SIC in $t_{2}$ and $Q_{\mathrm{Tu}_{1}}\left(\mathbf{w}_{21}, \mathbf{w}_{22}\right)$ is determined by

$$
Q_{\mathrm{Tu}_{1}}\left(\mathbf{w}_{21}, \mathbf{w}_{22}\right)=\frac{1}{2} \log _{2}\left(1+\rho_{1} g_{1}+\frac{\left|\mathbf{h}_{21}^{\dagger} \mathbf{w}_{21}\right|^{2}}{\left|\mathbf{h}_{21}^{\dagger} \mathbf{w}_{22}\right|^{2}+\sigma^{2}}\right) .
$$

Therefore, for this case, the subproblem P3-21 is represented by

$$
\begin{gathered}
\text { P3-21: } \max _{\mathbf{w}_{21}, \mathbf{w}_{22}} \alpha \log _{2}\left(1+\rho_{1} g_{1}+\frac{\left|\mathbf{h}_{21}^{\dagger} \mathbf{w}_{21}\right|^{2}}{\left|\mathbf{h}_{21}^{\dagger} \mathbf{w}_{22}\right|^{2}+\sigma^{2}}\right)+(1-\alpha) \log _{2}\left(1+\rho_{1} g_{1}\right) \\
\text { s.t. } \log _{2}\left(1+\rho_{1} g_{12}\right)<\log _{2}\left(1+\rho_{1} g_{1}+\frac{\left|\mathbf{h}_{21}^{\dagger} \mathbf{w}_{21}\right|^{2}}{\left|\mathbf{h}_{21}^{\dagger} \mathbf{w}_{22}\right|^{2}+\sigma^{2}}\right), \quad(47 \mathrm{a}) \\
\log _{2}\left(1+\rho_{1} \tilde{g}_{0}\right) \geq \log _{2}\left(1+\rho_{1} g_{1}+\frac{\left|\mathbf{h}_{21}^{\dagger} \mathbf{w}_{21}\right|^{2}}{\left|\mathbf{h}_{21}^{\dagger} \mathbf{w}_{22}\right|^{2}+\sigma^{2}}\right), \quad \text { (47c) } \\
\frac{1}{2} \log _{2}\left(1+\frac{\left|\mathbf{h}_{2}^{\dagger} \mathbf{w}_{22}\right|^{2}}{\left|\mathbf{h}_{2}^{\dagger} \mathbf{w}_{21}\right|^{2}+\sigma^{2}}\right) \geq Q, \\
\mathbf{w}_{21}^{\dagger} \mathbf{w}_{21}+\mathbf{w}_{22}^{\dagger} \mathbf{w}_{22} \leq P_{2},
\end{gathered}
$$

where the constraint 47b is the condition that $\mathrm{Ru}_{2}$ cannot apply SIC in $t_{2}$ and the constraint 47c is the condition to satisfy (46) referring to (40). Notice that constant $\frac{1}{2}$ is ignored in 47a without changing the property of the problem. From the observation of P3-21, the second term of the objective function, i.e., $(1-\alpha) \log _{2}\left(1+\rho_{1} g_{1}\right)$, is a constant, which can be ignored to obtain the solution. Then, we can see that for given $\alpha$, the right hand sides (RHSs) of constraints 47b and (47c) are equal to the objective function of 47a to be maximized, i.e., $\log _{2}\left(1+\rho_{1} g_{1}+\frac{\left|\mathbf{h}_{21}^{\dagger} \mathbf{w}_{21}\right|^{2}}{\left|\mathbf{h}_{21}^{\dagger} \mathbf{w}_{22}\right|^{2}+\sigma^{2}}\right)$. Hence, we note that if the problem $\mathbf{P 3}-\mathbf{2 1}$ is feasible, the constraint (47b) is always hold. Otherwise, P3 - 21 is infeasible and the solution is obtained by the other subproblems. In addition, if the constraint 47c is not hold, i.e., $\log _{2}\left(1+\rho_{1} \tilde{g}_{0}\right)<$ $\log _{2}\left(1+\rho_{1} g_{1}+\frac{\left|\mathbf{h}_{21}^{\dagger} \mathbf{w}_{21}\right|^{2}}{\left|\mathbf{h}_{21}^{\dagger} \mathbf{w}_{22}\right|^{2}+\sigma^{2}}\right)$, from (39), we observe that the expected achievable rate is bounded by constant, which is independent from $\mathbf{w}_{21}$ and $\mathbf{w}_{22}$. In other word, $\mathrm{Ru}_{1}$ can achieve at least constant rate in 39) if the constraint 47c is not hold. Hence, we can solve P3 - 21 without considering the constraint 47c) and after solving P3 - 21 without 47c), we can check whether the constraint $(47 \mathrm{c})$ is hold or not for the obtained solution. If (47c) is hold, the expected achievable rate at $\mathrm{Ru}_{1}$ is determined by the obtained solution and otherwise, the expected achievable rate is determined by the constant rate in (39). Therefore, by removing constraints (47b) and 47c), the subproblem $\mathbf{P 3}-\mathbf{2 1}$ can be equivalently rewritten as

$$
\begin{aligned}
\text { P3-21' }: \max _{\mathbf{w}_{21}, \mathbf{w}_{22}} & \frac{\left|\mathbf{h}_{21}^{\dagger} \mathbf{w}_{21}\right|^{2}}{\left|\mathbf{h}_{21}^{\dagger} \mathbf{w}_{22}\right|^{2}+\sigma^{2}} \\
\text { s.t. } & \left|\mathbf{h}_{2}^{\dagger} \mathbf{w}_{22}\right|^{2} \geq\left(4^{Q}-1\right)\left(\left|\mathbf{h}_{2}^{\dagger} \mathbf{w}_{21}\right|^{2}+\sigma^{2}\right), \\
& \mathbf{w}_{21}^{\dagger} \mathbf{w}_{21}+\mathbf{w}_{22}^{\dagger} \mathbf{w}_{22} \leq P_{2} .
\end{aligned}
$$

Since the problem $\mathbf{P} \mathbf{3}-\mathbf{2} \mathbf{1}^{\prime}$ is non-convex and $\mathbf{w}_{21}, \mathbf{w}_{22}$ are still coupled in the constraints, it is hard to directly obtain the solution of $\mathbf{P 3}-\mathbf{2 1 ^ { \prime }}$ in its current form. To solve $\mathbf{P 3}-\mathbf{2 1}$ by decoupling $\mathbf{w}_{21}$ and $\mathbf{w}_{22}$, we apply block coordinate update (BCU) method to update $\mathbf{w}_{21}$ (or $\mathbf{w}_{22}$ ) while fixing $\mathbf{w}_{22}$ (or $\left.\mathbf{w}_{21}\right)$ at one iteration, and optimize $\mathbf{w}_{22}\left(\right.$ or $\left.\mathbf{w}_{21}\right)$ based on the newly updated $\mathbf{w}_{21}$ ( or $\mathbf{w}_{22}$ ) at the next iteration. Thus, the expected achievable rate at $\mathrm{Ru}_{1}$ is maximized by optimizing $\mathbf{w}_{21}$ and $\mathbf{w}_{22}$ iteratively. Employing the semidefinite relaxation (SDR) technique [23] and giving $\mathbf{w}_{22}^{k}$ at $k+1$-th iteration, the relaxation of (48) can be rewritten as

$$
\begin{aligned}
\text { P3-21'-1: } \max _{\mathbf{W}_{21} \succeq \mathbf{0}} & \operatorname{tr}\left(\mathbf{W}_{21} \mathbf{h}_{21} \mathbf{h}_{21}^{\dagger}\right) \\
\text { s.t. }\left|\mathbf{h}_{2}^{\dagger} \mathbf{W}_{22}^{k}\right|^{2} \geq\left(4^{Q}-1\right)\left[\operatorname{tr}\left(\mathbf{W}_{21} \mathbf{h}_{2} \mathbf{h}_{2}^{\dagger}\right)+\sigma^{2}\right] & , \\
& \operatorname{tr}\left(\mathbf{W}_{21}\right)+\left(\mathbf{w}_{22}^{k}\right)^{\dagger} \mathbf{w}_{22}^{k} \leq P_{2}
\end{aligned}
$$

where we discard the constraint $\operatorname{rank}\left(\mathbf{W}_{21}\right)=1$. The relaxed problem 49) can be solved conveniently by existing solvers, such as CVX [24]. It is noted that the sufficient and necessary condition for the equivalence of problems $\mathbf{P} 3-\mathbf{2} \mathbf{1}^{\prime}-\mathbf{1}$ and $\mathbf{P} 3-\mathbf{2} \mathbf{1}^{\prime}$ with given $\mathbf{w}_{22}^{k}$, is that the optimal $\mathbf{W}_{21}^{*, k+1}$ obtained at $k+1$-th iteration of $\mathbf{P} \mathbf{3}-\mathbf{2} \mathbf{1}^{\prime} \mathbf{- 1}$ is rank-one, i.e, $\mathbf{W}_{21}^{*, k+1}=\mathbf{w}_{21}^{*, k+1}\left(\mathbf{w}_{21}^{*, k+1}\right)^{\dagger}$, which can be guaranteed by the following lemma.

Lemma 2: [25, Theorem 2.2] Let $\mathbf{A}_{i} \in \mathbb{C}^{n \times n}, i \in \mathcal{I}=$ $\{1,2,3\}$, be a Hermitian matrix and $\mathbf{X} \in \mathcal{H}_{+}^{n}$ be a nonzero 
TABLE I

The Proposed ALgorithm For SIMO

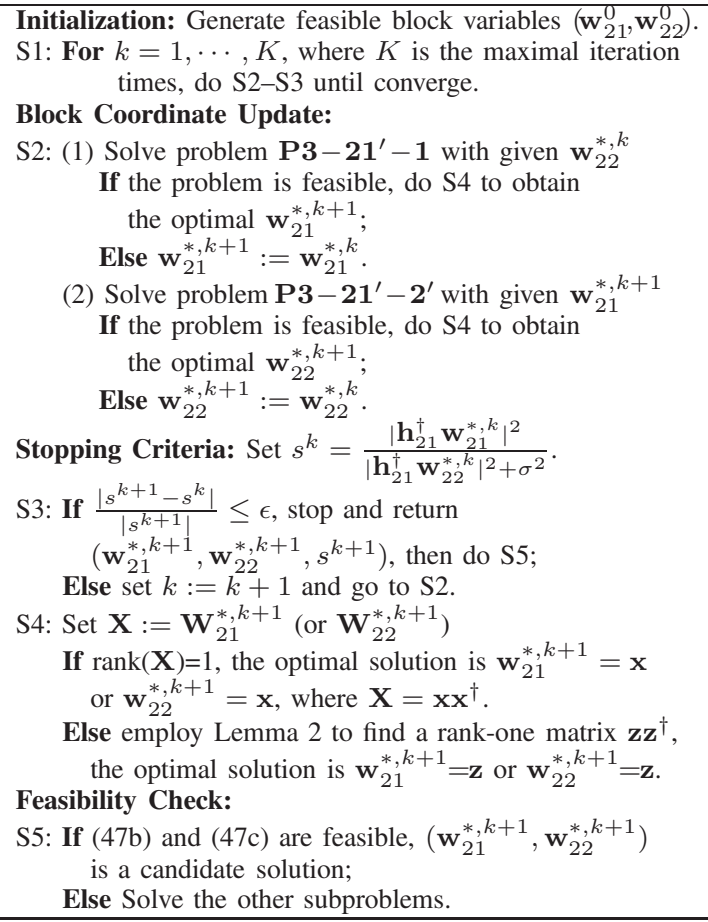

Hermitian positive semidefinite matrix. If $\operatorname{rank}(\mathbf{X}) \geq 2$, we can find a rank-one matrix $\mathbf{x x}^{\dagger}$ in polynomial-time such that $\operatorname{tr}\left(\mathbf{A}_{i} \mathbf{x} \mathbf{x}^{\dagger}\right)=\operatorname{tr}\left(\mathbf{A}_{i} \mathbf{X}\right), i \in \mathcal{I}$.

Proof: The proof of Lemma 2 can be referred to [25].

After achieving the rank-one solution $\mathbf{w}_{21}^{*, k+1}$ from $\mathbf{W}_{21}^{*, k+1}$ resorting to Lemma 2 if $\mathbf{P 3} \mathbf{- 2 \mathbf { 1 } ^ { \prime }}$ is feasible, we plug it into $\mathbf{P 3}-\mathbf{2} \mathbf{1}^{\prime}$. In a similar way to $\mathbf{P} 3-\mathbf{2} \mathbf{1}^{\prime}-\mathbf{1}$, for given $\mathbf{w}_{21}^{*, k+1}$, we solve the following problem

$$
\begin{aligned}
& \text { P3-21'-2: } \text { min }_{\mathbf{W}_{22} \succeq \mathbf{0}} \operatorname{tr}\left(\mathbf{W}_{22} \mathbf{h}_{21} \mathbf{h}_{21}^{\dagger}\right) \\
& \text { s.t.tr }\left(\mathbf{W}_{22} \mathbf{h}_{2} \mathbf{h}_{2}^{\dagger}\right) \geq\left(4^{Q}-1\right)\left(\left|\mathbf{h}_{2}^{\dagger} \mathbf{w}_{21}^{*, k+1}\right|^{2}+\sigma^{2}\right), \\
& \left(\mathbf{w}_{21}^{*, k+1}\right)^{\dagger} \mathbf{w}_{21}^{*, k+1}+\operatorname{tr}\left(\mathbf{W}_{22}\right) \leq P_{2} \text {. }
\end{aligned}
$$

Similarly, we can obtain the rank-one $\mathbf{w}_{22}^{*, k+1}$ from $\mathbf{W}_{22}^{*, k+1}$ at $k+1$-th iteration based on Lemma 2 if problem $\mathbf{P} \mathbf{3}-\mathbf{2 1} \mathbf{1}^{\prime}-\mathbf{2}$ is feasible. Consequently, we can iteratively obtain the optimal solution $\left(\mathbf{w}_{21}^{*}, \mathbf{w}_{22}^{*}\right)$ of $\mathbf{P 3}-\mathbf{2 1}^{\prime}$. Then, by using $\left(\mathbf{w}_{21}^{*}, \mathbf{w}_{22}^{*}\right)$, we can check the feasibility of the

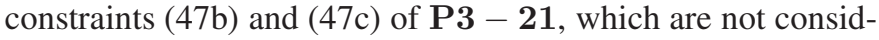
ered to obtain $\left(\mathbf{w}_{21}^{*}, \mathbf{w}_{22}^{*}\right)$. If the constraints are feasible, the obtained solution $\left(\mathbf{w}_{21}^{*}, \mathbf{w}_{22}^{*}\right)$ can be a candidate of the optimal beamformers for SIMO case, which are chosen among the solutions of four subproblems. Otherwise, the optimal solution is obtained by solving the other subproblems. The proposed iterative algorithm to solve $\mathbf{P 3}-\mathbf{2 1}$ is summarized in the TABLE I.

\section{MIMO case $\left(N_{1} \geq 2\right.$ and $\left.N_{2} \geq 2\right)$}

In this subsection, we consider that both $\mathrm{Tu}_{1}$ and $\mathrm{Tu}_{2}$ are equipped with $N_{1} \geq 2$ and $N_{2} \geq 2$ antennas and hence, the MIMO channel is formed between $\mathrm{Tu}_{1}$ and $\mathrm{Tu}_{2}$. For the MIMO case, we jointly design the transmit beamformer at $\mathrm{Tu}_{1}, \mathbf{w}_{1}$, and beamformers at $\mathrm{Tu}_{2}, \mathbf{w}_{21}$ and $\mathbf{w}_{22}$, to maximize the expected achievable rate at $\mathrm{Ru}_{1}$. In this case, the relaying transmission of $\mathrm{Tu}_{2}$ cannot improve the achievable rate if the channel quality between $\mathrm{Tu}_{1}$ and $\mathrm{Tu}_{2}$ is worse than the direct channel from $\mathrm{Tu}_{1}$ to $\mathrm{Ru}_{1}$ such as

$$
\begin{aligned}
\max _{\mathbf{W}_{1}} \log _{2}\left(1+\frac{\mathbf{w}_{1}^{\dagger} \mathbf{H}_{0}^{\dagger} \mathbf{H}_{0} \mathbf{w}_{1}}{\sigma^{2}}\right) & =\log _{2}\left(1+\rho_{1} \lambda_{\max }\left(\mathbf{H}_{0}^{\dagger} \mathbf{H}_{0}\right)\right) \\
& \leq \log _{2}\left(1+\rho_{1} \tilde{g}_{1}\right),
\end{aligned}
$$

where $\lambda_{\max }(\mathbf{X})$ is the largest eigenvalue of $\mathbf{X}$. Therefore, for the MIMO case, the expected achievable rate at $\mathrm{Ru}_{1}$ is given by

$R_{\mathrm{Ru}_{1}}\left(\mathbf{w}_{1}, \mathbf{w}_{21}, \mathbf{w}_{22}\right)=\left\{\begin{array}{l}\bar{R}_{\mathrm{Ru}_{1}}\left(\mathbf{w}_{1}, \mathbf{w}_{21}, \mathbf{w}_{22}\right), \text { if } \lambda_{\max }\left(\mathbf{H}_{0}^{\dagger} \mathbf{H}_{0}\right)>\tilde{g}_{1}, \\ \frac{1}{2} \log _{2}\left(1+\rho_{1} \tilde{g}_{1}\right), \text { otherwise },\end{array}\right.$

where $\bar{R}_{\mathrm{Ru}_{1}}\left(\mathbf{w}_{1}, \mathbf{w}_{21}, \mathbf{w}_{22}\right)$ is given by

$$
\begin{gathered}
\bar{R}_{\mathrm{Ru}_{1}}\left(\mathbf{w}_{1}, \mathbf{w}_{21}, \mathbf{w}_{22}\right)=\frac{\alpha}{2} \min \left[\log _{2}\left(1+\frac{\left|\mathbf{h}_{1}^{\dagger} \mathbf{w}_{1}\right|^{2}}{\sigma^{2}}+\frac{\left|\mathbf{h}_{21}^{\dagger} \mathbf{w}_{21}\right|^{2}}{\left|\mathbf{h}_{21}^{\dagger} \mathbf{w}_{22}\right|^{2}+\sigma^{2}}\right),\right. \\
\left.\log _{2}\left(1+\frac{\left\|\mathbf{H}_{0} \mathbf{w}_{1}\right\|^{2}}{\sigma^{2}}\right)\right]+\frac{1-\alpha}{2} \log _{2}\left(1+\frac{\left|\mathbf{h}_{1}^{\dagger} \mathbf{w}_{1}\right|^{2}}{\sigma^{2}}\right)
\end{gathered}
$$

We define the first term in (53) as

$$
\begin{aligned}
Q_{\mathrm{Tu}_{1}}\left(\mathbf{w}_{1}, \mathbf{w}_{21}, \mathbf{w}_{22}\right)= & \frac{1}{2} \min \left[\log _{2}\left(1+\frac{\left|\mathbf{h}_{1}^{\dagger} \mathbf{w}_{1}\right|^{2}}{\sigma^{2}}+\frac{\left|\mathbf{h}_{21}^{\dagger} \mathbf{w}_{21}\right|^{2}}{\left|\mathbf{h}_{21}^{\dagger} \mathbf{w}_{22}\right|^{2}+\sigma^{2}}\right),\right. \\
& \left.\log _{2}\left(1+\frac{\left\|\mathbf{H}_{0} \mathbf{w}_{1}\right\|^{2}}{\sigma^{2}}\right)\right]
\end{aligned}
$$

For the case that $\mathrm{Tu}_{2}$ helps the transmission of $\mathrm{Tu}_{1}$, if the rate achieved at $\mathrm{Ru}_{2}$ in $t_{1}, R_{12}\left(\mathbf{w}_{1}\right)$, is greater than $Q_{\mathrm{Tu}_{1}}\left(\mathbf{w}_{1}, \mathbf{w}_{21}, \mathbf{w}_{22}\right)$, i.e., $R_{12}\left(\mathbf{w}_{1}\right)=$ $\frac{1}{2} \log _{2}\left(1+\frac{\left|\mathbf{h}_{12}^{\dagger} \mathbf{w}_{1}\right|^{2}}{\sigma^{2}}\right) \geq Q_{\mathrm{Tu}_{1}}\left(\mathbf{w}_{1}, \mathbf{w}_{21}, \mathbf{w}_{22}\right), \mathrm{Ru}_{2}$ can decode $x_{1}$ in $t_{1}$ and thus, $\mathrm{Ru}_{2}$ can employ SIC to eliminate the effect of $x_{1}$ in its received signal in $t_{2}$. Therefore, the achievable rate at $\mathrm{Ru}_{2}$ is given by

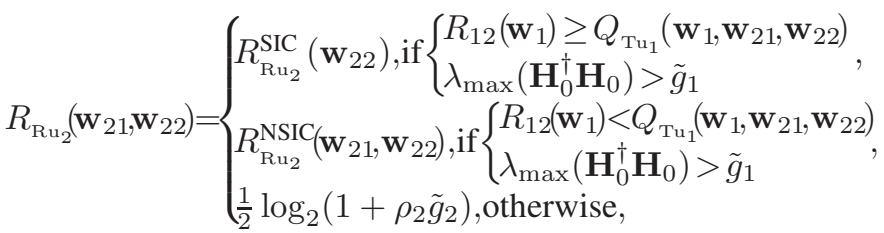

where $\tilde{g}_{2}=\left\|\mathbf{h}_{2}\right\|^{2}, R_{\mathrm{Ru}_{2}}^{\mathrm{SIC}}\left(\mathbf{w}_{22}\right)$ and $R_{\mathrm{Ru}_{2}}^{\mathrm{NSIC}_{2}}\left(\mathbf{w}_{21}, \mathbf{w}_{22}\right)$ are given in 42).

For the MIMO case, in order to maximize the expected achievable rate at $\mathrm{Ru}_{1}$ while guaranteeing QoS requirement at $\mathrm{Ru}_{2}$, the beamformer at $\mathrm{Tu}_{1}, \mathrm{w}_{1}$, and the beamformers at $\mathrm{Tu}_{2}$, $\mathbf{w}_{21}$ and $\mathbf{w}_{22}$, are jointly optimized by the following problem

$$
\begin{aligned}
\mathbf{P 4}: \max _{\mathbf{w}_{1}, \mathbf{w}_{21}, \mathbf{w}_{22}} & R_{\mathrm{Ru}_{1}}\left(\mathbf{w}_{1}, \mathbf{w}_{21}, \mathbf{w}_{22}\right) \\
\text { s.t. } & R_{\mathrm{Ru}_{2}}\left(\mathbf{w}_{21}, \mathbf{w}_{22}\right) \geq Q, \\
& \mathbf{w}_{1}^{\dagger} \mathbf{w}_{1} \leq P_{1}, \mathbf{w}_{21}^{\dagger} \mathbf{w}_{21}+\mathbf{w}_{22}^{\dagger} \mathbf{w}_{22} \leq P_{2},
\end{aligned}
$$


where $Q \in\left[0, Q^{\max }\right]$. Here, when $Q=Q^{\max }, \mathrm{Tu}_{2}$ does not have the residual power to help $\mathrm{Tu}_{1}$ and hence, we have $\mathbf{w}_{21}^{\dagger} \mathbf{w}_{21}=0$ and $\mathbf{w}_{22}^{\dagger} \mathbf{w}_{22}=P_{2}$. The power allocation at $\mathrm{Tu}_{2}$ is embedded in the beamformer design.

Similar with SIMO case, $\mathbf{P} 4$ in (56) can be divided into four subproblems with respect to whether $\mathrm{Ru}_{2}$ applies SIC, and the forms of $R_{\mathrm{Ru}_{1}}\left(\mathbf{w}_{1}, \mathbf{w}_{21}, \mathbf{w}_{22}\right)$. Due to the space limitation and the similarities of the subproblems in MIMO case, in the following, we consider the subproblem $\mathbf{P} \mathbf{4}-\mathbf{2 1}$, where $\mathrm{Ru}_{2}$ does not apply SIC in $t_{2}$ and $Q_{\mathrm{Tu}_{1}}\left(\mathbf{w}_{1}, \mathbf{w}_{21}, \mathbf{w}_{22}\right)$ is determined by

$$
Q_{\mathrm{Tu}_{1}}\left(\mathbf{w}_{1}, \mathbf{w}_{21}, \mathbf{w}_{22}\right)=\frac{1}{2} \log _{2}\left(1+\frac{\left|\mathbf{h}_{1}^{\dagger} \mathbf{w}_{1}\right|^{2}}{\sigma^{2}}+\frac{\left|\mathbf{h}_{21}^{\dagger} \mathbf{w}_{21}\right|^{2}}{\left|\mathbf{h}_{21}^{\dagger} \mathbf{w}_{22}\right|^{2}+\sigma^{2}}\right) .
$$

Therefore, for this case, the subproblem P3-21 is represented by

P4-21: $\max _{\mathbf{\mathbf { w } _ { 1 }}, \mathbf{w}_{21}, \mathbf{w}_{22}} \alpha \log _{2}\left(1+\frac{\left|\mathbf{h}_{1}^{\dagger} \mathbf{w}_{1}\right|^{2}}{\sigma^{2}}+\frac{\left|\mathbf{h}_{21}^{\dagger} \mathbf{w}_{21}\right|^{2}}{\left|\mathbf{h}_{21}^{\dagger} \mathbf{w}_{22}\right|^{2}+\sigma^{2}}\right)+(1-\alpha) \log _{2}\left(1+\frac{\left|\mathbf{h}_{1}^{\dagger} \mathbf{w}_{1}\right|^{2}}{\sigma^{2}}\right)$

$$
\text { s.t. } \log _{2}\left(1+\frac{\left|\mathbf{h}_{12}^{\dagger} \mathbf{w}_{1}\right|^{2}}{\sigma^{2}}\right)<\log _{2}\left(1+\frac{\left|\mathbf{h}_{1}^{\dagger} \mathbf{w}_{1}\right|^{2}}{\sigma^{2}}+\frac{\left|\mathbf{h}_{21}^{\dagger} \mathbf{w}_{21}\right|^{2}}{\left|\mathbf{h}_{21}^{\dagger} \mathbf{w}_{22}\right|^{2}+\sigma^{2}}\right),
$$

$$
\log _{2}\left(1+\frac{\left\|\mathbf{H}_{0} \mathbf{w}_{1}\right\|^{2}}{\sigma^{2}}\right) \geq \log _{2}\left(1+\frac{\left|\mathbf{h}_{1}^{\dagger} \mathbf{w}_{1}\right|^{2}}{\sigma^{2}}+\frac{\left|\mathbf{h}_{21}^{\dagger} \mathbf{w}_{21}\right|^{2}}{\left|\mathbf{h}_{21}^{\dagger} \mathbf{w}_{22}\right|^{2}+\sigma^{2}}\right),
$$

$$
\begin{aligned}
& \frac{1}{2} \log _{2}\left(1+\frac{\left|\mathbf{h}_{2}^{\dagger} \mathbf{w}_{22}\right|^{2}}{\left|\mathbf{h}_{2}^{\dagger} \mathbf{w}_{21}\right|^{2}+\sigma^{2}}\right) \geq Q, \\
& \mathbf{w}_{1}^{\dagger} \mathbf{w}_{1} \leq P_{1}, \mathbf{w}_{21}^{\dagger} \mathbf{w}_{21}+\mathbf{w}_{22}^{\dagger} \mathbf{w}_{22} \leq P_{2},
\end{aligned}
$$

where the constraint 58b is the condition that $\mathrm{Ru}_{2}$ cannot apply SIC in $t_{2}$ and the constraint (58c) is the condition that (54) becomes (57). Since $\mathbf{P} \mathbf{4}-\mathbf{2 1}$ is non-convex and $\mathbf{w}_{1}, \mathbf{w}_{21}$ and $\mathbf{w}_{22}$ are coupled in the constraints, it is hard to directly obtain the solution of $\mathbf{P} 4-\mathbf{2 1}$.

In order to obtain the beamformers for MIMO case, we combine the optimal beamformer structure of MISO case, which is given in Lemma 1 to obtain $\mathbf{w}_{1}$, and the BCU based iterative algorithm for SIMO case, which is given in TABLE I, to obtain $\mathbf{w}_{21}$ and $\mathbf{w}_{22}$. At one iteration, for given $\mathbf{w}_{21}$ and $\mathbf{w}_{22}$, we obtain $\mathbf{w}_{1}$ based on the optimal structure in 29 . Then, at the next iteration, $\mathbf{w}_{21}$ and $\mathbf{w}_{22}$ are optimized for the fixed $\mathbf{w}_{1}$ by using BCU based algorithm to maximize the expected achievable rate.

For given $\left(\mathbf{w}_{21}, \mathbf{w}_{22}\right)$, the terms related to $\left(\mathbf{w}_{21}, \mathbf{w}_{22}\right)$ can be regarded as the constant and then, we can see that the problem $\mathbf{P} 4-\mathbf{2 1}$ has the same structure of the problem for MISO case, which optimizes $\mathbf{w}_{1}$ only. Hence, for given $\left(\mathbf{w}_{21}, \mathbf{w}_{22}\right)$, we design $\mathbf{w}_{1}$ based on the optimal structure of the beamformer for MISO, given in (29). The optimal beamformer for MISO is constructed by two bases $\mathbf{w}_{0}=$ $\frac{\boldsymbol{\Pi}_{\mathbf{h}_{0}} \mathbf{h}_{1}}{\left\|\boldsymbol{\Pi}_{\mathbf{h}_{0}} \mathbf{h}_{1}\right\|}$ and $\mathbf{w}_{0}^{\perp}=\frac{\boldsymbol{\Pi}_{\mathbf{h}_{0}}^{\perp} \mathbf{h}_{1}}{\left\|\boldsymbol{\Pi}_{\mathbf{h}_{0}}^{\perp} \mathbf{h}_{1}\right\|}$. Here, since $\mathbf{h}_{0}=\theta \boldsymbol{\Pi}_{\mathbf{h}_{0}} \mathbf{h}_{1}$ for some scalar $\theta$ and $\mathbf{h}_{1}=\boldsymbol{\Pi}_{\mathbf{h}_{0}} \mathbf{h}_{1}+\boldsymbol{\Pi}_{\mathbf{h}_{0}}^{\perp} \mathbf{h}_{1}$, the optimal
TABLE II

The Proposed Algorithm For MIMO

Initialization: Define $\lambda_{m}=\frac{m}{M}, m=1, \cdots, M$, where $M$
is a positive integer. Generate feasible block
variables $\left(\mathbf{w}_{21}^{0}, \mathbf{w}_{22}^{0}\right)$.
Repeat: For each given $\lambda_{m}$, set
$\mathbf{w}_{1}:=\mathbf{w}_{1}\left(\lambda_{m}\right)=\sqrt{P_{1}} \frac{\lambda_{m} \mathbf{w}_{0}^{\text {eig }}+\left(1-\lambda_{m}\right) \mathbf{w}_{1}^{\text {mrt }}}{\left\|\lambda_{m} \mathbf{w}_{0}^{\text {eig }}+\left(1-\lambda_{m}\right) \mathbf{w}_{1}^{\text {mrt }}\right\|}$, and do
$\mathrm{S} 1-\mathrm{S} 3$ to search for $\left(\mathbf{w}_{21,}^{*}, \lambda_{m}, \mathbf{w}_{22, \lambda_{m}}^{*}\right)$ related with $\lambda_{m}$.
$\mathrm{S} 1:$ For $k=1, \cdots, K$, where $K$ is the maximal iteration
times, do the following until converge.
S2: Solve problem P4-21 without constraints 58b 58c
based on S2-S5 in TABLE I.

beamformer for MISO in 29) can be represented by two bases $\mathbf{w}_{0}^{\mathrm{mrt}}=\frac{\mathbf{h}_{0}}{\left\|\mathbf{h}_{0}\right\|}$ and $\mathbf{w}_{1}^{\mathrm{mrt}}=\frac{\mathbf{h}_{1}}{\left\|\mathbf{h}_{1}\right\|}$ such as

$$
\mathbf{w}_{1}^{\mathrm{opt}}=\lambda_{1} \mathbf{w}_{0}^{\mathrm{mrt}}+\lambda_{2} \mathbf{w}_{1}^{\mathrm{mrt}}
$$

where $\lambda_{1}$ and $\lambda_{2}$ are determined to satisfy $\left\|\mathbf{w}_{1}^{\mathrm{opt}}\right\|^{2}=1$. From (59), we note that the optimal structure of the beamformer for MISO is the linear combination of MRT beamformers of channels $\mathbf{h}_{0}$ and $\mathbf{h}_{1}$. Thus, for MIMO case, we design $\mathbf{w}_{1}$ based on the beamformer structure in (59) as

$$
\mathbf{w}_{1}(\lambda)=\sqrt{P_{1}} \frac{\lambda \mathbf{w}_{0}^{\text {eig }}+(1-\lambda) \mathbf{w}_{1}^{\mathrm{mrt}}}{\left\|\lambda \mathbf{w}_{0}^{\text {eig }}+(1-\lambda) \mathbf{w}_{1}^{\mathrm{mrt}}\right\|},
$$

where $\mathbf{w}_{0}^{\text {eig }}$ is an eigenvector corresponding the largest eigenvalue of $\mathbf{H}_{0}^{\dagger} \mathbf{H}_{0}$ and $\lambda$ is a real value in $0 \leq \lambda \leq 1$. For the MIMO channel, the eigenvector corresponding the largest eigenvalue of $\mathbf{H}_{0}^{\dagger} \mathbf{H}_{0}$ is the beamformer that maximizes the achievable rate of $\mathbf{H}_{0}$, similar to MRT beamformer for MISO channel. Based on (60), for given $\left(\mathbf{w}_{21}, \mathbf{w}_{22}\right)$, we optimize the coefficient $\lambda$ to maximize the expected achievable rate at $\mathrm{Ru}_{1}$ by one-dimensional line search.

Similarly, for given $\mathbf{w}_{1}$, the terms related to $\mathbf{w}_{1}$ can be regarded as the constant and thus, the problem $\mathbf{P 4 - 2 1}$ becomes the problem that has the same structure of that for SIMO case, which optimizes $\mathbf{w}_{21}$ and $\mathbf{w}_{22}$ only. Therefore, for given $\mathbf{w}_{1}$, we can obtain $\mathbf{w}_{21}$ and $\mathbf{w}_{22}$ by using the BCU based iterative algorithm for SIMO, proposed in Section III-C. Consequently, for MIMO case, we obtain the beamforers $\mathbf{w}_{1}$, $\mathbf{w}_{21}$ and $\mathbf{w}_{22}$ iteratively to maximize the expected achievable rate at $\mathrm{Ru}_{1}$ while guaranteeing the $\mathrm{QoS}$ of $\mathrm{Ru}_{2}$. The details of the proposed algorithm for MIMO case are summarized in TABLE II.

\section{Simulation Results}

In this section, we evaluate the performance of the trust degree based user cooperation for three cases: SISO case where all users have a single antenna $\left(N_{1}=N_{2}=1\right)$, MISO case where $\mathrm{Tu}_{1}$ has the multiple antennas $\left(N_{1}=\right.$ 2, $N_{2}=1$ ), SIMO case where $\mathrm{Tu}_{2}$ has the multiple antennas $\left(N_{1}=1, N_{2}=2\right)$ and MIMO case where both $\mathrm{Tu}_{1}$ and $\mathrm{Tu}_{2}$ have the multiple antennas $\left(N_{1}=\right.$ $N_{2}=2$ ). Unless otherwise specified, we use the average gains of channel elements as $\left\{\sigma_{H_{0}}^{2}, \sigma_{h_{1}}^{2}, \sigma_{h_{2}}^{2}, \sigma_{h_{12}}^{2}, \sigma_{h_{21}}^{2}\right\}=$ $\{-35,-45,-30,-25,-25\} \mathrm{dB}$ and the expected achievable rates are averaged over $10^{4}$ channel realizations. 


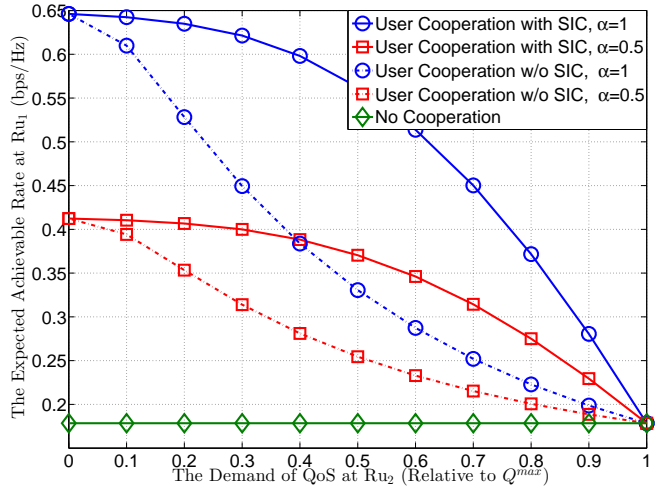

Fig. 2. The expected achievable rate at $\mathrm{Ru}_{1}$ versus the $\mathrm{QoS}$ requirement at $\mathrm{Ru}_{2}$, where $\rho_{1}=\rho_{2}=40 \mathrm{~dB}$ and $N_{1}=N_{2}=1$.

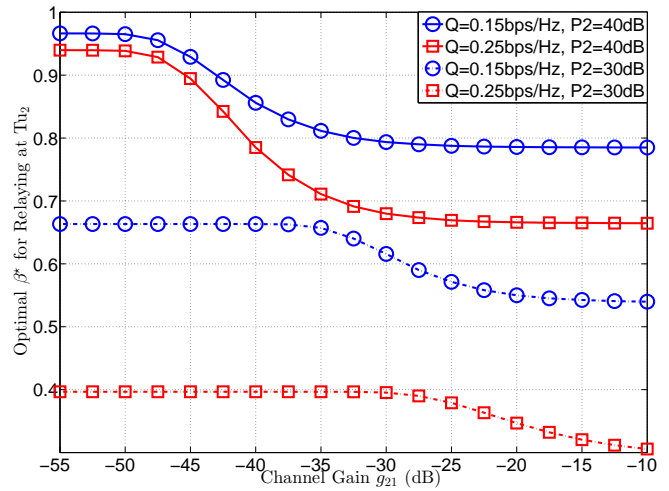

Fig. 3. Optimal $\beta^{\star}$ at $\mathrm{Tu}_{2}$ versus channel gain $g_{21}$ with different given QoS and $P_{2}, \alpha=0.5, \rho_{1}=40 \mathrm{~dB}$ and $N_{1}=N_{2}=1$.

\section{A. SISO case $\left(N_{1}=N_{2}=1\right)$}

In Fig 2, for SISO case, we plot the expected achievable rates of $\mathrm{Ru}_{1}$ according to the QoS requirement at $\mathrm{Ru}_{2}, Q$, when the transmit SNR at $\mathrm{Tu}_{1}$ and $\mathrm{Tu}_{2}$ are given by $\rho_{1}=\rho_{2}=$ $40 \mathrm{~dB}$. To compare with the proposed user cooperation scheme, which applies SIC, we also plot the expected achievable rates of the user cooperation without SIC and no cooperation $(\alpha=0)$. For the proposed user cooperation, the optimal power allocation at $\mathrm{Tu}_{2}$ for cooperation is obtained as $\beta^{\star}$ in Theorem 1 In Fig 2 we show that the expected achievable rate can be significantly increased by the user cooperation when the trust degree between users is high such as $\alpha=1$. For the case with $\alpha=1, \mathrm{Tu}_{2}$ always helps the transmission of $\mathrm{Tu}_{1}$ while when $\alpha=0.5, \mathrm{Tu}_{2}$ helps the transmission of $\mathrm{Tu}_{1}$ with probability 0.5 even if $\mathrm{Tu}_{2}$ has sufficient power budget after achieving QoS. In addition, when the QoS requirement at $\mathrm{Ru}_{2}$ is small, $\mathrm{Tu}_{1}$ can achieve very high expected achievable rate because $\mathrm{Tu}_{2}$ has a large amount of residual power after achieving its $\mathrm{QoS}$ and helps the transmission of $\mathrm{Tu}_{1}$ by using large power. By applying SIC at $\mathrm{Ru}_{2}$, the expected achievable rate at $\mathrm{Ru}_{1}$ can be further improved since $\mathrm{Tu}_{2}$ can achieve QoS requirement with small power, and hence $\mathrm{Tu}_{2}$ can allocate more power for cooperation than that without SIC.

In Fig 3, for different $Q(Q=0.5$ and $0.3 \mathrm{bps} / \mathrm{Hz})$ and $\rho_{2}\left(\rho_{2}=40\right.$ and $\left.30 \mathrm{~dB}\right)$, the optimal power allocation for cooperation at $\mathrm{Tu}_{2}, \beta^{\star}$, is plotted as a function of the

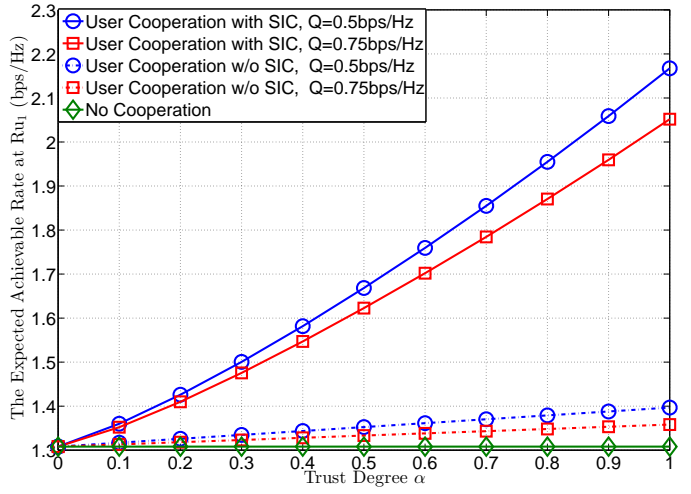

Fig. 4. The expected achievable rate at $\mathrm{Ru}_{1}$ versus trust degree $\alpha$, where $\rho_{1}=\rho_{2}=50 \mathrm{~dB}$ and $N_{1}=2, N_{2}=1$.

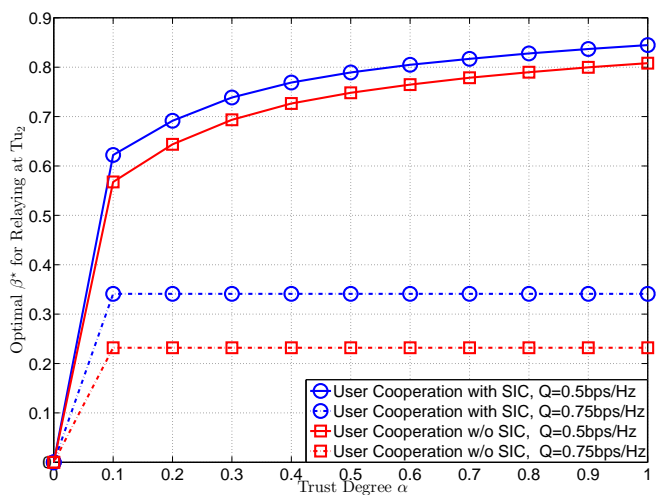

Fig. 5. Optimal $\beta^{\star}$ for relaying at Tu 2 versus trust degree $\alpha$, where $\rho_{1}=$ $\rho_{2}=50 \mathrm{~dB}$ and $N_{1}=2, N_{2}=1$.

channel gain from $\mathrm{Tu}_{2}$ to $\mathrm{Ru}_{1}, g_{21}$, when $\alpha=0.5$ and $\rho_{1}=40 \mathrm{~dB}$. In this figure, we use the channel gains as $\left\{\sigma_{H_{0}}^{2}, \sigma_{h_{1}}^{2}, \sigma_{h_{2}}^{2}, \sigma_{h_{12}}^{2}\right\}=\{-32,-40,-30,-32\} \mathrm{dB}$. When $g_{21}$ is weak, from (12), $\mathrm{Ru}_{2}$ can apply SIC and $\mathrm{Tu}_{2}$ can allocate more power for cooperation. Hence, the optimal power allocation is obtained by $\beta^{\star}=\beta_{Q_{1}}$. In contrast, when $g_{21}$ is strong, $\mathrm{Tu}_{2}$ cannot allocate large power for cooperation and the optimal power allocation is obtained by $\beta^{\star}=\beta_{Q_{2}}$. Otherwise, $\mathrm{Tu}_{2}$ reduces $\beta$ to help applying SIC at $\mathrm{Ru}_{1}$, and the optimal power allocation is determined by $\beta^{\star}=\tilde{\beta}_{1}$, which is a decreasing function of $g_{21}$. In addition, we can see that $\mathrm{Tu}_{2}$ can allocate more power for cooperation when the QoS requirement at $\mathrm{Ru}_{2}, Q$, is small or the amount of transmit power budget at $\mathrm{Tu}_{2}$ is large.

\section{B. $\operatorname{MISO}$ case $\left(N_{1}=2, N_{2}=1\right)$}

In the MISO case, we jointly design the beamformer at $\mathrm{Tu}_{1}, \mathbf{w}_{1}$, and the power allocation for cooperation at $\mathrm{Tu}_{2}, \beta$, according to trust degree, $\alpha$. For the proposed user cooperation, the beamformer at $\mathrm{Tu}_{1}$ is obtained by Theorem 2 and the corresponding power allocation is obtained by one dimensional search. For the comparison, we show the performance of the case that $\mathrm{Tu}_{1}$ simply uses a MRT beamformer, $\mathbf{w}_{\mathbf{1}}=\mathbf{w}_{1}^{\mathrm{mrt}}=$ $\frac{\mathbf{h}_{1}}{\left\|\mathbf{h}_{1}\right\|}$, and $\mathrm{Ru}_{2}$ does not apply SIC. The performance of the no cooperation between users $(\alpha=0)$ with MRT beamformer is also given as a baseline. 
In Fig. 4 for the proposed and reference schemes, we plot the expected achievable rates of $\mathrm{Ru}_{1}$ according to trust degree, $\alpha$ when $\rho_{1}=\rho_{2}=50 \mathrm{~dB}$. With the growth of $\alpha$, for the user cooperation schemes, the expected achievable rates of $\mathrm{Ru}_{1}$ are increased by the cooperation of $\mathrm{Tu}_{2}$. For the proposed user cooperation, since the beamformer is efficiently designed by considering both trust degree and physical channel qualities, the performance improvement becomes significantly large according to $\alpha$. However, when $\mathrm{Tu}_{1}$ uses MRT beamformer, the expected achievable rate improvement is marginal because beamformer is designed independently from $\alpha$ and hence, the benefit from the cooperation cannot be fully exploited even the trust degree is high.

Fig. 5, the optimal power allocation for cooperation at $\mathrm{Tu}_{2}$, $\beta^{\star}$, is plotted according to $\alpha$. When the $\mathrm{Tu}_{1}$ transmits its data with MRT beamformer, which is independently designed from $\alpha$, the corresponding power allocation to maximize the expected achievable rate is also determined independently as a constant. For the proposed beamforming, in Fig. 5, we can see that $\beta^{\star}$ increases with the growth of trust degree $\alpha$. In the proposed beamforming, when $\alpha$ is high, the direction of beamformer is steered from $\mathbf{h}_{1}$ to $\mathbf{h}_{0}$ to fully utilize the cooperation of $\mathrm{Tu}_{2}$. Due to constraint of $\mathrm{DF}$ relaying, the expected achievable rate in (24) is maximized by balancing the minimum rates achieved at $\mathrm{Ru}_{1}$ (first term) and $\mathrm{Tu}_{2}$ (second term). Hence, for high $\alpha$, (24) is maximized by increasing the second term from beamforming design and compensating the first term by assigning large power for cooperation at $\mathrm{Tu}_{2}$, i.e., $\beta^{\star}$ is high.

C. SIMO case $\left(N_{1}=1, N_{2}=2\right)$ and MIMO case $\left(N_{1}=\right.$ $2, N_{2}=2$ )

For the SIMO case, we evaluate the performance of the proposed user cooperation based on trust degree in terms of the expected achievable rate. The expected achievable rate at the proposed scheme is achieved by beamformers obtained from the proposed algorithm, which is given in TABLE I. In Fig. 6 for $Q=0.5$ and $1 \mathrm{bps} / \mathrm{Hz}$, we plot the expected achievable rates of the proposed and reference schemes versus the trust degree, $\alpha$ when $\rho_{1}=\rho_{2}=50 \mathrm{~dB}$. Similar to previous subsections, we can see that for high $\alpha$, the expected achievable rate at $\mathrm{Ru}_{1}$ is significantly increased by the cooperation with $\mathrm{Tu}_{2}$ and the performance is further improved by efficiently designing beamformers based on the proposed algorithm. For the MIMO case, we can see the similar phenomena for $Q=1$ and $2 \mathrm{bps} / \mathrm{Hz}$ in Fig. 8

In Fig. 7, for $Q=0.5$ and $1 \mathrm{bps} / \mathrm{Hz}$, the expected achievable rate at $\mathrm{Ru}_{1}$ is plotted according to the relaying channel quality from $\mathrm{Tu}_{2}$ to $\mathrm{Ru}_{1}, \tilde{g}_{21}=\left\|\mathbf{h}_{21}\right\|^{2}$. In this figure, the trust degree and transmit SNRs are $\alpha=0.5$ and $\rho_{1}=\rho_{2}=50 \mathrm{~dB}$. From Fig. 7 we first observe that when the gain of relaying channel increases from $-50 \mathrm{~dB}$ to $-25 \mathrm{~dB}$, the expected achievable rate at $\mathrm{Ru}_{1}$ can be increased by cooperative transmission from $\mathrm{Tu}_{2}$ via $\mathbf{h}_{21}$. However, the expected achievable rate does not increase and is saturated when $\tilde{g}_{21}$ increases in the regime of $\tilde{g}_{21}>-25 \mathrm{~dB}$. Since when the quality of the relaying channel, $\mathbf{h}_{21}$, is much better than that of channel between $\mathrm{Tu}_{1}$ and

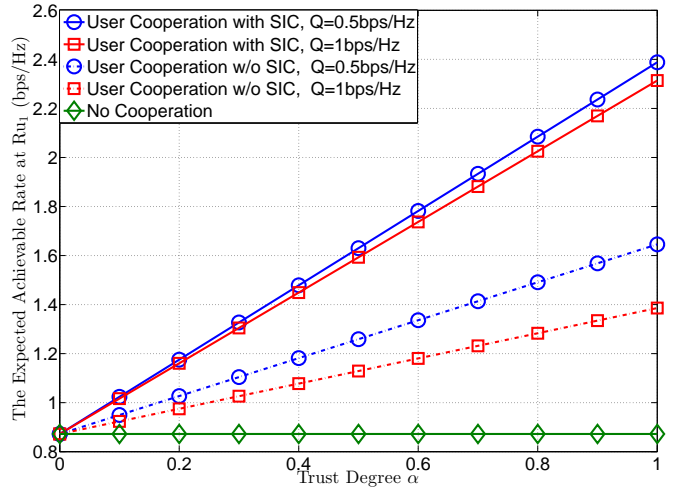

Fig. 6. The expected achievable rate at $\mathrm{Ru}_{1}$ versus trust degree $\alpha$, where $\rho_{1}=\rho_{2}=50 \mathrm{~dB}$ and $N_{1}=1, N_{2}=2$.

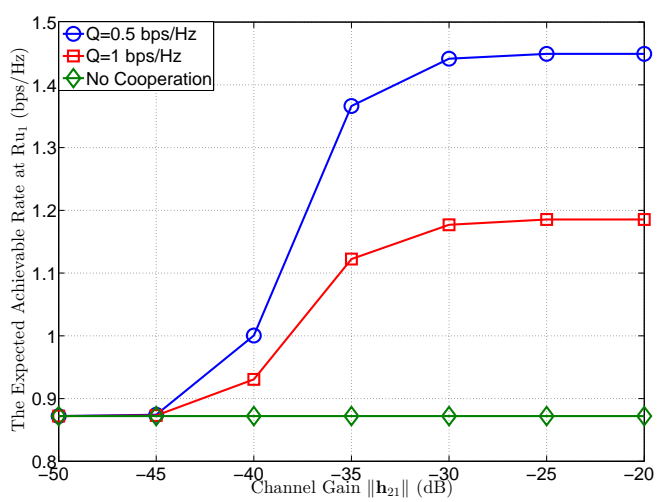

Fig. 7. The expected achievable rate at $\mathrm{Ru}_{1}$ versus channel gain $\left\|\mathbf{h}_{21}\right\|^{2}$ with respect to different given QoS, where $\rho_{1}=\rho_{2}=50 \mathrm{~dB}, N_{1}=1, N_{2}=2$ and $\alpha=0.5$.

$\mathrm{Tu}_{2}, \mathbf{h}_{0}$, due to the DF relaying constraint, the rate achieved at $\mathrm{Tu}_{2}$, which is the second term of (40), is always lower than the rate achieved at $\mathrm{Ru}_{1}$, which is the first term of (40) for all feasible beamformers. Hence, the expected achievable rate cannot increase and is saturated even if the quality of the relaying channel is sufficiently good. For the MIMO case, we can see the similar phenomena for $Q=1$ and $2 \mathrm{bps} / \mathrm{Hz}$ in Fig. 9

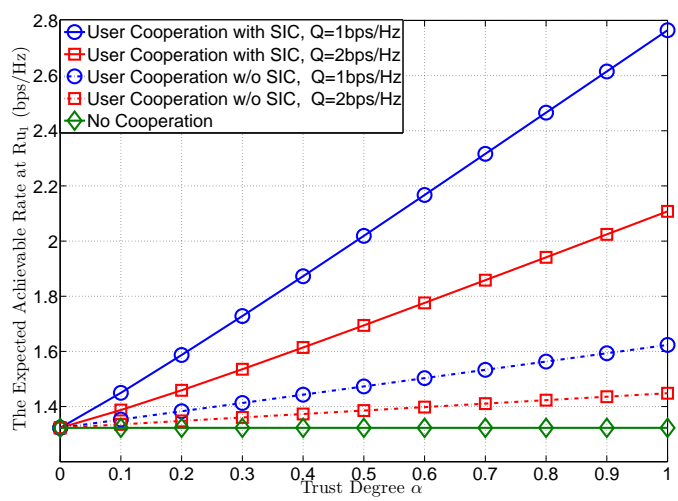

Fig. 8. The expected achievable rate at $\mathrm{Ru}_{1}$ versus trust degree $\alpha$, where $\rho_{1}=\rho_{2}=50 \mathrm{~dB}$ and $N_{1}=N_{2}=2$. 


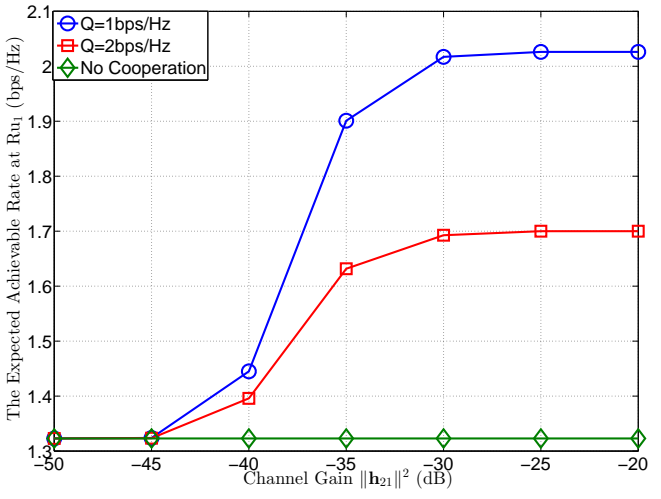

Fig. 9. The expected achievable rate at $\mathrm{Ru}_{1}$ versus channel gain $\left\|\mathbf{h}_{21}\right\|^{2}$ with different given QoS, where $\rho_{1}=\rho_{2}=50 \mathrm{~dB}, N_{1}=N_{2}=2$ and $\alpha=0.5$.

\section{CONClusion}

In this paper, we propose the user cooperation techniques in the multiple antenna system with two communication pairs, i.e., $\mathrm{Tu}_{1}-\mathrm{Ru}_{1}$ and $\mathrm{Tu}_{2}-\mathrm{Ru}_{2}$, where $\mathrm{Tu}_{2}$ can help the transmission of $\mathrm{Tu}_{1}$ according to the trust degree. For different antenna configurations at $\mathrm{Tu}_{1}$ and $\mathrm{Tu}_{2}$, we design the user cooperation strategies by taking into account the trust degree information as well as channel information. For the SISO case, as a special case, we first propose an optimal power allocation strategy at $\mathrm{Tu}_{2}$, which maximizes the expected achievable rate at $\mathrm{Ru}_{1}$ while guaranteeing $\mathrm{QoS}$ requirement at $\mathrm{Ru}_{2}$, according to the channel qualities and QoS requirement. For the MISO case, we provide an optimal structure of beamformer as a linear combination of the weighted channel vectors. Then, based on the optimal structure, we obtain the beamformer that maximizes an approximated expected achievable rate as a function of the trust degree and corresponding power allocation at $\mathrm{Tu}_{2}$. For the SIMO case, to jointly optimize the beamformers of $\mathrm{Tu}_{2}$, we utilize semidefinite relaxation (SDR) technique and block coordinate update (BCU) method to solve the considered problem, and guarantee the rank-one solutions at each step. Furthermore, for the MIMO case, the similarities among problem structures related to MISO, SIMO and MIMO cases inspire us to combine the design of beamformer at $\mathrm{Tu}_{1}$ from MISO and the alternative algorithm from SIMO together to jointly optimize the beamformers at $\mathrm{Tu}_{1}$ and $\mathrm{Tu}_{2}$ to maximize the expected achievable rate at $\mathrm{Ru}_{1}$. Finally, we show that the trust degree between users can be used to significantly improve the expected achievable rate in the user cooperation networks.

\section{APPENDIX A}

\section{PROOF OF THEOREM 1}

From (11), we note that the expected achievable rate at $\mathrm{Ru}_{1}, R_{\mathrm{Ru}_{1}}(\beta)$, is an increasing function with $\beta$. Hence, the optimal power allocation is determined by the maximum $\beta$ that satisfies the QoS requirement at $\mathrm{Ru}_{2}$ such as $R_{\mathrm{Ru}_{2}}(\beta) \geq Q$. If SIC can be applied at $\mathrm{Ru}_{2}, R_{\mathrm{Ru}_{2}}(\beta)$ is given by $R_{\mathrm{Ru}_{2}}^{\mathrm{SIC}}(\beta)$ and otherwise, $R_{R u_{2}}(\beta)$ is given by $R_{\mathrm{Ru}_{2}}^{\mathrm{NSIC}}(\beta)$. In order to find maximum $\beta$ that satisfies $R_{\mathrm{Ru}_{2}}(\beta) \geq Q$, we first find the conditions that SIC can be applied at $\mathrm{Ru}_{2}$ and for these conditions, we find the optimal $\beta$.

From (13), $Q_{\mathrm{Tu}_{1}}(\beta)$ can be represented by two cases of $\beta_{0} \leq \beta \leq 1$ and $0 \leq \beta \leq \beta_{0}$. First, for the case of $\beta_{0} \leq \beta \leq$ $1, Q_{\mathrm{Tu}_{1}}(\beta)$ is given by

$$
Q_{\mathrm{Tu}_{1}}=\frac{1}{2} \log _{2}\left(1+\rho_{1} g_{0}\right),
$$

which is a constant and independent from $\beta$. For this case, if $g_{12} \geq g_{0}$, we have $R_{12} \geq Q_{\mathrm{Tu}_{1}}$ and thus, $\mathrm{Ru}_{2}$ can apply SIC. To satisfy QoS requirement at $\mathrm{Ru}_{2}$, we obtain the condition of $\beta$ as

$$
R_{\mathrm{Ru}_{2}}^{\mathrm{SIC}}(\beta) \geq Q \Rightarrow \beta \leq \beta_{Q_{1}},
$$

where $\beta_{Q_{1}}$ is given in (18). In this case, the optimal $\beta$ is feasible as $\beta^{\star}=\beta_{Q_{1}}$ if $\beta_{0} \leq \beta_{Q_{1}}$, and thus, the condition of $Q$ that makes $\beta^{\star}$ feasible can be obtained by

$$
\beta_{0} \leq \beta_{Q_{1}} \Rightarrow Q \leq r_{1},
$$

where $r_{1}$ is given in (21). Therefore, if $g_{12} \geq g_{0}$ and $Q \leq r_{1}$, the optimal $\beta$ is obtained by $\beta^{\star}=\beta_{Q_{1}}$ and $\mathrm{QoS}$ of $\mathrm{Ru}_{2}$ is achieved by applying SIC at $\mathrm{Ru}_{2}$.

For the case of $0 \leq \beta \leq \beta_{0}, Q_{\mathrm{Tu}_{1}}(\beta)$ is given by

$$
Q_{\mathrm{Tu}_{1}}(\beta)=\frac{1}{2} \log _{2}\left(1+\rho_{1} g_{1}+\frac{\beta \rho_{2} g_{21}}{(1-\beta) \rho_{2} g_{21}+1}\right) .
$$

For this case, by using (A.4), the condition that can apply SIC at $\mathrm{Ru}_{2}$ is obtained by

$$
R_{12}=\frac{1}{2} \log \left(1+\rho_{1} g_{12}\right) \geq Q_{\mathrm{Tu}_{1}}(\beta) \Rightarrow g_{12} \geq g_{1}, \beta \leq \tilde{\beta}_{1},
$$

where $\tilde{\beta}_{1}$ is given in 20 . Thus, if $\beta_{Q_{1}} \leq \beta_{0}$ and $\beta_{Q_{1}} \leq \tilde{\beta}_{1}$, the optimal $\beta$ is obtained by $\beta^{\star}=\beta_{Q_{1}}$ because $\beta_{Q_{1}}$ is the maximum $\beta$ that satisfies the $\mathrm{QoS}$ requirement at $\mathrm{Ru}_{2}$ as given in A.2. We obtain the condition of $Q$ that satisfies $\beta_{Q_{1}} \leq \beta_{0}$ and $\beta_{Q_{1}} \leq \tilde{\beta}_{1}$ as

$$
\left\{\begin{array}{l}
\beta_{Q_{1}} \leq \beta_{0} \Rightarrow Q \geq r_{1}, \\
\beta_{Q_{1}} \leq \tilde{\beta}_{1} \Rightarrow Q \geq r_{2},
\end{array} \Rightarrow Q \geq \max \left(r_{1}, r_{2}\right),\right.
$$

where $r_{2}$ is given in 21). Therefore, if $g_{12} \geq g_{1}$ and $Q \geq$ $\max \left(r_{1}, r_{2}\right)$, the optimal $\beta$ is obtained by $\beta^{\star}=\beta_{Q_{1}}$ and QoS requirement at $\mathrm{Ru}_{2}$ is achieved by applying SIC at $\mathrm{Ru}_{2}$.

On the other hand, if $\tilde{\beta}_{1} \leq \beta_{Q_{1}}$, equivalent $Q \leq r_{2}$, we cannot guarantee the $\mathrm{QoS}$ requirement at $\mathrm{Ru}_{2}$ by $\beta=\beta_{Q_{1}}$ because $\mathrm{Ru}_{2}$ cannot apply SIC when $\beta=\beta_{Q_{1}}$. Hence, for $Q \leq r_{2}$, the $\mathrm{QoS}$ requirement at $\mathrm{Ru}_{2}$ can be guaranteed by $\beta=\tilde{\beta}_{1}$ with applying SIC or $\beta=\beta_{Q_{2}}$ without applying SIC. Here, $\beta_{Q_{2}}$ is obtained to satisfy QoS requirement at $\mathrm{Ru}_{2}$ without using SIC as $R_{\mathrm{Ru}_{2}}^{\mathrm{NSIC}}\left(\beta_{Q_{2}}\right)=Q$.

For $Q \leq r_{2}$, since if $\beta_{0}>\tilde{\beta}_{1}>\beta_{Q_{2}}$, the optimal $\beta$ to guarantee $\mathrm{QoS}$ requirement at $\mathrm{Ru}_{2}$ is given by $\beta^{\star}=\tilde{\beta}_{1}$, the condition that makes $\beta^{\star}=\tilde{\beta}_{1}$ is obtained as

$$
\left\{\begin{array}{l}
\tilde{\beta}_{1}<\beta_{0} \quad \Rightarrow g_{0}>g_{12}, \\
\tilde{\beta}_{1}>\beta_{Q_{2}} \Rightarrow Q>r_{3},
\end{array}\right.
$$

where $r_{3}$ is given in 21). Therefore, if $g_{0}>g_{12} \geq g_{1}$ and $r_{2} \geq Q>r_{3}$, the optimal $\beta$ is obtained by $\beta^{\star}=\tilde{\beta}_{1}$ and QoS requirement at $\mathrm{Ru}_{2}$ is achieved by applying SIC at $\mathrm{Ru}_{2}$.

Otherwise, the optimal $\beta$ is obtained by $\beta^{\star}=\beta_{Q_{2}}$ and QoS requirement at $\mathrm{Ru}_{2}$ is achieved without applying SIC at $\mathrm{Ru}_{2}$. 


\section{APPENDIX B \\ Proof of THEOREM 2}

For given $\beta$, by substituting $\mathbf{w}_{1}^{\text {opt }}$ of Lemma 1 into (28), $\tilde{R}_{\mathrm{Ru}_{1}}\left(\mathbf{w}_{1}\right)$ can be rewritten by

$$
\begin{aligned}
\tilde{R}_{\mathrm{Ru}_{1}}(\eta)= & \frac{\alpha}{2} \log _{2}\left\{\rho_{1} \min \left(g(\eta)+m_{1}(\beta), f(\eta)\right)\right\} \\
& +\frac{1-\alpha}{2} \log _{2}\left(\rho_{1} g(\eta)\right),
\end{aligned}
$$

where $g(\eta)$ and $f(\eta)$ are given by

$$
\begin{aligned}
& g(\eta) \triangleq\left(\sqrt{\eta v_{1}}+\sqrt{(1-\eta) v_{2}}\right)^{2}=\left|\mathbf{h}_{1}^{\dagger} \mathbf{w}_{1}\right|^{2}, \\
& f(\eta) \triangleq \eta \tilde{g}_{0}=\left|\mathbf{h}_{0}^{\dagger} \mathbf{w}_{1}\right|^{2} .
\end{aligned}
$$

First, if $\tilde{g}_{0}$ is large as $\tilde{g}_{0}>\frac{\left(v_{1}+v_{2}\right)^{2}}{v_{1}}$ and power allocation for cooperation, $\beta$, is small as $\beta<\underline{\beta}$, we have $f(\eta)>g(\eta)+m_{1}(\beta)$ for any $\eta$ in $\frac{v_{1}}{v_{1}+v_{2}} \leq \eta \leq 1$ and thus, $\tilde{R}_{\mathrm{Ru}_{1}}(\eta)$ in B.1) can be given as

$$
\tilde{R}_{\mathrm{Ru}_{1}}(\eta)=\frac{\alpha}{2} \log _{2}\left\{\rho_{1}\left(g(\eta)+m_{1}(\beta)\right)\right\}+\frac{1-\alpha}{2} \log _{2}\left(\rho_{1} g(\eta)\right) .
$$

Since (B.4) is a decreasing function with $\eta$ in $\frac{v_{1}}{v_{1}+v_{2}} \leq \eta \leq 1$, we can obtain $\eta^{\star}$ that maximize (B.4) as $\eta^{\star}=\frac{v_{1}}{v_{1}+v_{2}}$.

Contrarily, if $\tilde{g}_{0}$ is small as $\tilde{g}_{0}<v_{1}$ or $\beta$ is large as $\beta>\bar{\beta}$ for $\tilde{g}_{0} \geq v_{1}$, we have $f(\eta)<g(\eta)+m_{1}(\beta)$ for any $\eta$ in $\frac{v_{1}}{v_{1}+v_{2}} \leq \eta \leq 1$. In this case, B.1 can be rewritten as

$$
\tilde{R}_{\mathrm{Ru}_{1}}(\eta)=\frac{\alpha}{2} \log _{2}\left(\rho_{1} f(\eta)\right)+\frac{1-\alpha}{2} \log _{2}\left(\rho_{1} g(\eta)\right) .
$$

Since $(\mathbb{B} .5$ is a concave function with respect to $\eta$, we obtain $\eta^{\star}$ to maximize (B.5) by solving $\frac{\partial \tilde{R}_{\mathrm{Ru}_{1}}(\eta)}{\partial \eta}=0$ as $\eta^{\star}=\eta_{2}$, which is given in (30).

Otherwise, according to $\beta, \tilde{R}_{\mathrm{Ru}_{1}}(\eta)$ in (B.1) can be represented by either B.4 or B.5. We first derive $\eta_{3}(\beta)$, which is given in (32), to satisfy $f\left(\eta_{3}(\beta)\right)=g\left(\eta_{3}(\beta)\right)+m_{1}(\beta)$. Then, for $\eta_{3}(\beta) \leq \eta \leq 1, \tilde{R}_{\mathrm{Ru}_{1}}(\eta)$ is represented by B.4 , which is a decreasing function of $\eta$ and thus, we can obtain $\eta^{\star}=\eta_{3}(\beta)$, given in (30). For $\frac{v_{1}}{v_{1}+v_{2}} \leq \eta \leq \eta_{3}(\beta), \tilde{R}_{\mathrm{Ru}_{1}}(\eta)$ is represented by (B.5), which is a concave function of $\eta$ achieving maximum value at $\eta^{\star}=\eta_{2}$. Therefore, if $\eta_{2}$ is in the range of $\frac{v_{1}}{v_{1}+v_{2}} \leq \eta \leq \eta_{3}(\beta)$, we can obtain $\eta^{\star}=\eta_{2}$. Otherwise, if $\eta_{2}>\eta_{3}(\beta)$, we obtain $\eta^{\star}=\eta_{3}(\beta)$ because (B.5) is an increasing function of $\eta$ for $\frac{v_{1}}{v_{1}+v_{2}} \leq \eta \leq \eta_{3}(\beta)$. Consequently, if $\eta_{2} \leq \eta_{3}(\beta)$, we obtain the optimal $\eta$ as $\eta^{\star}=\eta_{2}$, and otherwise, the optimal $\eta$ is obtained by $\eta^{\star}=\eta_{3}(\beta)$. Therefore, the optimal $\eta$ can be represented by $\eta^{\star}=\min \left\{\eta_{2}, \eta_{3}(\beta)\right\}$.

\section{REFERENCES}

[1] Q. Li, K. H. Li, and K. C. Teh, "Diversity-multiplexing tradeoff of wireless communication systems with user cooperation," IEEE Trans. Inf. Theory, vol. 57, no. 9, pp. 5794-5819, 2011.

[2] R. A. Loodaricheh, S. Mallick, and V. K. Bhargava, "Energy-efficient resource allocation for OFDMA cellular networks with user cooperation and QoS provisioning," IEEE Trans. Wireless Commun., vol. 13, no. 11, pp. 6132-6146, 2014.

[3] M. Nokleby and B. Aazhang, "User cooperation for energy-efficient cellular communications," in Proc. IEEE ICC, 2010.

[4] G. Zhang, K. Yang, P. Liu, and J. Wei, "Power allocation for full-duplex relaying based D2D communication underlaying cellular networks," IEEE Trans. Veh. Tech., vol. 64, no. 10, pp. 4911-4916, 2015.
[5] D. Hwang, D. I. Kim, S. K. Choi, and T.-J. Lee, "UE relaying cooperation over D2D uplink in heterogeneous cellular networks," IEEE Trans. Commun., vol. 63, no. 12, pp. 4784-4796, 2015.

[6] A. Radwan, M. Albano, J. Rodriguez, and C. Verikoukis, "Analysis of energy saving using cooperation use-case: Wifi and wimedia," in Proc. IEEE FutureNetw, 2012, pp. 1-10.

[7] Y. Chen and S. Kishore, "A game-theoretic analysis of Decode-andForward user cooperation," IEEE Trans. Wireless Commun., vol. 7, no. 5, pp. 1941-1951, 2008.

[8] J. P. Coon, "Modelling trust in random wireless networks," in Proc. IEEE ISWCS, 2014

[9] X. Gong, X. Chen, and J. Zhang, "Social group utility maximization game with applications in mobile social networks," in Proc. IEEE Allerton, 2013.

[10] — - "Social group utility maximization in mobile networks: From altruistic to malicious behavior," in Proc. IEEE CISS, 2014, pp. 1-6.

[11] X. Chen, B. Proulx, X. Gong, and J. Zhang, "Exploiting social ties for cooperative D2D communications: a mobile social networking case," IEEE Trans. Wireless Commun., vol. 23, no. 5, pp. 1471-1484, 2015.

[12] Y. Li, T. Wu, P. Hui, D. Jin, and S. Chen, "Social-aware D2D communications: qualitative insights and quantitative analysis," IEEE Commun. Mag., vol. 52, no. 6, pp. 150-158, 2014.

[13] Y. Zhang, L. Song, W. Saad, Z. Dawy, and Z. Han, "Exploring social ties for enhanced device-to-device communications in wireless networks," in Proc. IEEE GLOBECOM, 2013.

[14] M. Zhang, X. Chen, and J. Zhang, "Social-aware relay selection for cooperative networking: An optimal stopping approach," in Proc. IEEE ICC, 2014.

[15] J. Y. Ryu, J. Lee, and T. Q. Quek, "Trust degree based beamforming for miso cooperative communication system," IEEE Commun. Lett., vol. 19, no. 11 , pp. 1957-1960, 2015.

[16] _ - "Confidential cooperative communication with trust degree of potential eavesdroppers," IEEE Trans. Wireless Commun., vol. 15, no. 6, pp. 3823-3836, 2016.

[17] J. Li, R. Li, and J. Kato, "Future trust management framework for mobile ad hoc networks," IEEE Commun. Mag., vol. 46, no. 4, pp. 108-114, 2008.

[18] Y. L. Sun, Z. Han, W. Yu, and K. R. Liu, "A trust evaluation framework in distributed networks: Vulnerability analysis and defense against attacks." in Proc. IEEE INFOCOM, vol. 2006, pp. 1-13.

[19] G. Theodorakopoulos and J. S. Baras, "On trust models and trust evaluation metrics for ad hoc networks," IEEE J. Sel. Areas Commun., vol. 24, no. 2, pp. 318-328, 2006.

[20] C. Zouridaki, B. L. Mark, M. Hejmo, and R. K. Thomans, "A quantitative trust establishment framework for reliable data packet delivery in MANETs," in Proc. ACM Workshop on Security of Ad Hoc and Sensor Networks, 2005, pp. 1-10.

[21] R. Changiz, H. Halabian, F. R. Yu, I. Lambadaris, H. Tang, and P. C. Mason, "Trust establishment in cooperative wireless networks," in Proc. IEEE MILCOM, 2010, pp. 1074-1079.

[22] J. N. Laneman, D. N. Tse, and G. W. Wornell, "Cooperative diversity in wireless networks: Efficient protocols and outage behavior," IEEE Trans. Inf. Theory, vol. 50, no. 12, pp. 3062-3080, 2004.

[23] Z. Luo, W. Ma, A. M. So, Y. Ye, and S. Zhang, "Semidefinite relaxation of quadratic optimization problems," IEEE Signal Process. Mag., vol. 27, no. 3, pp. 20-34, 2010.

[24] M. Grant and S. Boyd, "CVX: Matlab software for Disciplined Convex Programming, Version 1.21," [Online]. Available: http://cvxr.com/cvx/.

[25] W. Ai, Y. Huang, and S. Zhang, "New results on hermitian matrix rankone decomposition." Mathematical Programming, vol. 128, no. 1-2, pp. 253-283, 2011. 


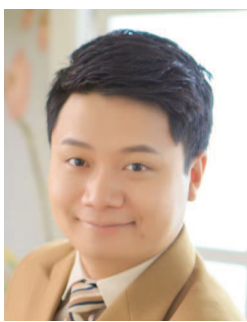

Mingxiong Zhao received the B.S. degree in Electrical Engineering and the Ph.D. degree in Information and Communication Engineering from South China University of Technology (SCUT), Guangzhou, China, in 2011 and 2016, respectively. He was a visiting Ph.D. student at University of Minnesota (UMN), Twin Cities, MN, USA, from 2012 to 2013 and Singapore University of Technology and Design (SUTD), Singapore, from 2015 to 2016, respectively. Since 2016, he has been an Assistant Professor at the School of Software, Yunnan University, Kunming, China. His current research interests are physical layer security, cooperative relay communication, and social aware communication systems.

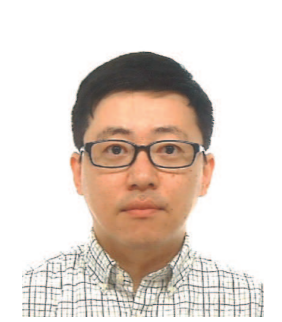

Jong Yeol Ryu (S'11-M'14) received the B.E. degree in Electrical Engineering from Chungnam National University, Daejeon, Korea, in 2008. He received the M.S. and Ph.D. degrees in Electrical Engineering from Korea Advance Institute of Science and Technology (KAIST), Daejeon, Korea, in 2010 and 2014, respectively. From 2014 to 2016, he was a Postdoctoral Fellow at Singapore University of Technology and Design (SUTD). Currently, he is an Assistant Professor at the Department of Information and Communication Engineering, Gyeongsang National University (GNU), Tongyeong, Korea. His current research interests are communication secrecy, cooperative user relay communications, and social aware communication systems.

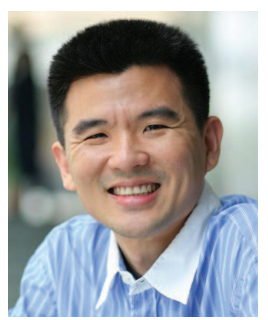

Tony Q.S. Quek (S'98-M'08-SM'12) received the B.E. and M.E. degrees in Electrical and Electronics Engineering from Tokyo Institute of Technology, respectively. At MIT, he earned the Ph.D. in Electrical Engineering and Computer Science. Currently, he is a tenured Associate Professor with the Singapore University of Technology and Design (SUTD). He also serves as the Associate Head of ISTD Pillar and the Deputy Director of the SUTD-ZJU IDEA. His main research interests are the application of mathematical, optimization, and statistical theories to communication, networking, signal processing, and resource allocation problems. Specific current research topics include heterogeneous networks, wireless security, internet-of-things, and big data processing.

Dr. Quek has been actively involved in organizing and chairing sessions, and has served as a member of the Technical Program Committee as well as symposium chairs in a number of international conferences. He is serving as the Workshop Chair for IEEE Globecom in 2017, the Tutorial Chair for the IEEE ICCC in 2017, and the Special Session Chair for IEEE SPAWC in 2017. $\mathrm{He}$ is currently an elected member of IEEE Signal Processing Society SPCOM Technical Committee. He was an Executive Editorial Committee Member for the IEEE TRANSACTIONS ON WIRELESS COMMUNICATIONS, an Editor for the IEEE TRANSACTIONS ON COMMUNICATIONS, and an Editor for the IEEE WiRELESS COMMUNiCATIONS LETTERS. He is a co-author of the book "Small Cell Networks: Deployment, PHY Techniques, and Resource Allocation" published by Cambridge University Press in 2013 and the book "Cloud Radio Access Networks: Principles, Technologies, and Applications" by Cambridge University Press in 2017.

Dr. Quek was honored with the 2008 Philip Yeo Prize for Outstanding Achievement in Research, the IEEE Globecom 2010 Best Paper Award, the 2012 IEEE William R. Bennett Prize, the IEEE SPAWC 2013 Best Student Paper Award, the IEEE WCSP 2014 Best Paper Award, the 2015 SUTD Outstanding Education Awards - Excellence in Research, the 2016 Thomson Reuters Highly Cited Researcher, and the 2016 IEEE Signal Processing Society Young Author Best Paper Award.

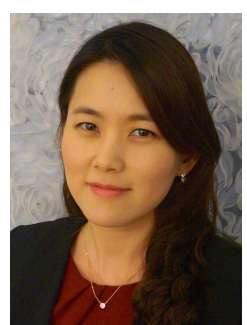

Jemin Lee (S'06-M'11) received the B.S. (with high honors), M.S., and Ph.D. degrees in Electrical and Electronic Engineering from Yonsei University, Seoul, Korea, in 2004, 2007, and 2010, respectively. She was a Postdoctoral Fellow at the Massachusetts Institute of Technology (MIT), Cambridge, MA from 2010 to 2013, and a Temasek Research Fellow at iTrust, Centre for Research in Cyber Security, Singapore University of Technology and Design (SUTD), Singapore from 2014 to 2016. Currently, she is an Assistant Professor at the Department of Information and Communication Engineering, Daegu Gyeongbuk Institute of Science and Technology (DGIST), Daegu, Korea. Her current research interests include physical layer security, wireless security, heterogeneous networks, and machine-type communication.

Dr. Lee is currently an Editor for the IEEE TRANSACTIONS ON WIRELESS COMMUNICATIONS and the IEEE COMMUNICATIONS LETTERS, and served as a Guest Editor for the IEEE WIRELESS COMMUNICATIONS, special issue on LTE in Unlicensed Spectrum, 2016, and the ELSEVIER PHYSICAL Communication, special issues on Physical Layer Security in 2016 and Heterogeneous and Small Cell Networks in 2014. She received the IEEE ComSoc Asia-Pacific Outstanding Young Researcher Award in 2014, the Temasek Research Fellowship in 2013, the Chun-Gang Outstanding Research Award in 2011, and the IEEE WCSP Best Paper Award in 2014.

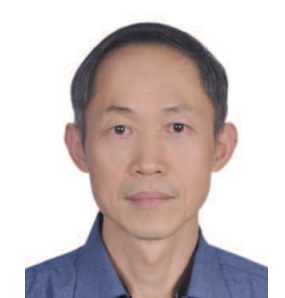

Suili Feng (M'05) received the B.S. degree in Electrical Engineering and the M.S. and Ph.D. degrees in Electronic and Communication System from South China University of Technology, Guangzhou, China, in 1982, 1989, and 1998, respectively. He was a Research Assistant with Hong Kong Polytechnic University, Hong Kong, from 1991 to 1992, and a Visiting Scholar with University of South Florida, Tampa, FL, USA, from 1998 to 1999 . He has been with South China University of Technology since 1989, where he is currently a Professor with the School of Electronic and Information Engineering. His research interests include wireless networks, computer networks, and communication signal processing. 